\title{
Multifragmentation, fragment flow, and the nuclear equation of state
}

\author{
G. Peilert, H. Stöcker, and W. Greiner \\ Institut für Theoretische Physik, Johann Wolfgang Goethe-Universität, \\ D-6000 Frankfurt am Main, Federal Republic of Germany
}

A. Rosenhauer

Gesellschaft für Schwerionenforschung, Postfach 110552, D-6100 Darmstadt, Federal Republic of Germany

A. Bohnet and J. Aichelin

Institut für Theoretische Physik, Universität Heidelberg and Max-Planck-Institut für Kernphysik, D-6900 Heidelberg, Federal Republic of Germany

(Received 11 April 1988; revised manuscript received 17 October 1988)

\begin{abstract}
The quantum molecular dynamic method is used to study multifragmentation and fragment flow and their dependence on in-medium cross sections, momentum dependent interactions, and the nuclear equation of state, for collisions of ${ }^{197} \mathrm{Au}+{ }^{197} \mathrm{Au}$ and ${ }^{93} \mathrm{Nb}+{ }^{93} \mathrm{Nb}$ in the bombarding energy regime from 100 to $800 \mathrm{~A} \mathrm{MeV}$. Time and impact parameter dependence of the fragment formation and their implications for the conjectured liquid-vapor phase transition are investigated. We find that the inclusive fragment mass distribution is independent of the equation of state and exhibits a power-law behavior $Y(A) \sim A^{-\tau}$ with an exponent $\tau \approx-2.3$. True multifragmentation events are found in central collisions for energies $E_{\text {lab }} \sim 30-200 \mathrm{MeV} /$ nucleon. The associated light fragment $(d, t, \alpha)$ to proton ratios increase with the multiplicity of charged particles and decrease with energy, in agreement with recent experiments. The calculated absolute charged particle multiplicities, the multiplicities of intermediate mass $(A>4)$ fragments, and their respective rapidity distributions do compare well with recent $4 \pi$ data, but are quite insensitive to the equation of state. On the other hand, these quantities depend sensitively on the nucleon-nucleon scattering cross section, and can be used to determine $\sigma$ experimentally. The transverse momentum flow of the complex fragments increases with the stiffness of the equation of state. Reduced (in-medium) $n-n$ scattering cross sections reduce the fragment flow. Momentum dependent interactions increase the fragment flow. It is shown that the measured fragment flow at $200 \mathrm{~A} \mathrm{MeV}$ can be reproduced in the model. We find that also the increase of the $p_{x} / A$ values with the fragment mass is in agreement with experiments. The calculated fragment flow is too small as compared to the plastic ball data, if a soft equation of state with in-medium corrections (momentum dependent interactions plus reduced cross sections) is employed. An alternative, most intriguing resolution of the puzzle about the stiffness of the equation of state could be an increase of the scattering cross sections due to precritical scattering in the vicinity of a phase transition.
\end{abstract}

\section{INTRODUCTION}

It has recently been argued ${ }^{1,2}$ that powerful prompt supernova explosions can only occur if the nuclear equation of state (EOS) is substantially softer at high densities than anticipated. This is in striking contrast to the analysis of the high-energy heavy-ion collision data.

Exclusive $4 \pi$ measurements ${ }^{3-9}$ established the collective flow, predicted by the nuclear fluid dynamics model. ${ }^{10-13}$ The absolute magnitude of the measured flow is surprisingly large, providing evidence for a stiff nuclear equation of state. ${ }^{13-17}$ In one of the last plastic ball experiments it has been shown that intermediate mass fragments up to $Z=10$ are abundantly produced in $\mathrm{Au}$ $(200 \mathrm{~A} \mathrm{MeV})+\mathrm{Au}$ reactions and that they exhibit even stronger flow effects. ${ }^{18,19}$

Here we study the fragment formation in heavy symmetric systems within the microscopic quantum molecular dynamic (QMD) theory. ${ }^{20-27}$ We investigate how the fragments are formed in such reactions. We study their energy and target mass dependence as well as their dependence on in-medium effects like a reduced hard core scattering cross section, momentum dependent interactions, and the nuclear EOS.

We demonstrate that the QMD model predicts the observed power law dependence of the inclusive fragment yields and that the predicted multifragmentation into $\approx 10$ complex $(A>4)$ fragments as well as the observed multiplicity and bombarding energy dependence of light ( $A=1-4$ ) fragment formation agrees with the data. The fragment multiplicities do not depend sensitively on the EOS.

The study of the fragment flow yields evidence for a stiff EOS. This apparent discrepancy between the "soft" EOS claimed to be necessary to yield supernovae explosions and the stiff EOS which reproduces the flow data could have various origins as follows.

(1) The iron core of the progenitor star could be small$\mathrm{er}^{28}$ than estimated previously. ${ }^{29}$ Then prompt supernovae explosions would occur even with a stiff EOS. ${ }^{30}$ 
(2) The supernovae explosions could be due to mechanisms others than the prompt bounce, e.g., by late shock revival due to neutrino heating. ${ }^{31}$

(3) The time scales involved in the two processes are quite different $\left(10^{-3} \mathrm{sec}\right.$ versus $\left.10^{-22} \mathrm{sec}\right)$, so that a softening of the neutron matter equation of state due to processes in $\beta$ equilibrium could play a role. ${ }^{32}$

(4) The angular momentum of the progenitor, which should play an important role in the prompt collapse, has been ignored in most calculations. ${ }^{1,2}$

(5) The momentum dependent interactions could provide an additional repulsion in heavy-ion collisions, which could help in producing the large observed transverse momentum transfer. ${ }^{33-35}$

(6) The in-medium scattering cross sections could be larger than the Uehling-Uhlenbeck (UU) cross section ${ }^{36}$ (possibly due to precritical scattering in the vicinity of a phase transition). ${ }^{37}$ This would be a most fascinating resolution of the puzzle about the stiffness of the EOS. However, recent calculations of the medium corrected scattering cross sections yield reduced values of $\sigma^{\text {eff }} \approx 0.7 \sigma^{\mathrm{UU}} .65,66$

Several microscopic models have been developed to describe heavy-ion collisions. The Vlasov-Uehling Uhlenbeck (VUU) model ${ }^{15-17,38-44}$ describes successfully single particle observables by evolving the semiclassical Wigner function in phase space (Pauli blocking and stochastic scattering are incorporated into this approach).

This model is intrinsically unable to describe cluster formation, which requires $A$ body correlations. Hence, up to now, only schematic or phenomenological models have been available. However, there has been considerable recent interest in the production of intermediate mass fragments $(A>4)$ in high energy protonnucleus $^{45-48}$ and heavy-ion collisions. ${ }^{48-56}$ The mass distributions of inclusive measurements exhibit a power-law form which has been interpreted as indication for a liquid-vapor phase transition. ${ }^{45-49}$

Other microscopic models are the molecular dynamics $^{33,57,58,14}$ approach, which can be useful to describe fragmentation in a classical way, ${ }^{59-61}$ the time dependent Hartree-Fock equations ${ }^{62}$ and other semiclassical models. ${ }^{63,64}$

Recently we advanced an extension to the classical molecular dynamics model, dubbed quantum molecular dynamics (QMD). This model simulates heavy ion reactions on an event-by-event basis and, as a consequence, preserves $N$-body correlations and fluctuations. A brief description of this model is given in Sec. II. A detailed description of our model is contained in Ref. 26, where the fragmentation of cold target spectator matter in asymmetric $\mathrm{Ne}(1050 \mathrm{~A} \mathrm{MeV})+\mathrm{Au}$ collisions is investigated. The interplay between the in-medium effects and the EOS on nuclear stopping and flow is studied in Refs. 24 and 27. A short account of the present work can be found in Ref. 23.

The inclusion of some important nonequilibrium aspects, e.g., the momentum dependent interactions (MDI), ${ }^{22}$ and in-medium effects via reduced nucleonnucleon scattering cross sections, ${ }^{24-27}$ are also given in Sec. II.
In Sec. III we present the results of the QMD calculations. The formation of complex fragments and their time evolution in configuration space in the reactions $\mathrm{Au}+\mathrm{Au}$ and $\mathrm{Nb}+\mathrm{Nb}$ in the energy interval from 30 to $800 \mathrm{~A} \mathrm{MeV}$ are discussed.

Then we study the impact parameter and bombarding energy dependence of the light ( $A=1-4)$ and intermediate mass $(A>4)$ fragment multiplicities. Because of the numerical expenditure of the gold on gold collisions we study the behavior of the light fragments in the lighter system $\mathrm{Nb}+\mathrm{Nb}$ at $100,250,400$, and $800 A \mathrm{MeV}$ bombarding energy.

In Sec. IV we switch back to the $\mathrm{Au}+\mathrm{Au}$ system to compare the predictions for the flow of the fragments with the data. The dependence of the flow on the EOS and the in-medium effects is discussed. The conclusions are given in Sec. $\mathrm{V}$.

\section{THE MODEL}

Neglecting all quantum features, the QMD model reduces to the classical molecular dynamics method $^{14,33,57-61}$ corresponding to the numerical solution of the $A_{T}+A_{P}$ Liouville equation. However, there are important quantum features which have to be respected: The nucleons have a finite width in configuration and momentum space. Collisions among nucleons are suppressed when they bring nucleons in already occupied or partially occupied phase space regions. Furthermore, the scattering amplitude does not relate the scattering angle with the impact parameter in a unique way. Rather, the square of the scattering amplitude can be identified with a probability distribution. The scattering angle as well as the Pauli blocking of collisions are treated statistically. The nucleons are represented by Wigner densities of Gaussian wave packets of the form

$$
f_{i}(\mathbf{r}, \mathbf{p}, t)=\left(\frac{1}{\pi \hbar}\right)^{3} e^{-\left[\mathbf{p}-\mathbf{p}_{0 i}(t)\right]^{2} L^{2} / \hbar^{2}} e^{-\left[\mathbf{r}-\mathbf{r}_{0 i}(t)\right]^{2} / L^{2}} .
$$

Those wave packets obey the uncertainty relation $\Delta x \cdot \Delta p_{x}=\hbar / 2 . \quad \mathbf{r}_{0 i}$ and $\mathbf{p}_{0 i}$ are the centroids of the wave packet $i$ in phase space, which evolve in time, whereas the widths $L$ of the wave packets are kept fixed.

Our model will be extended to include the time dependence of this width parameter. This can, e.g., be done by representing each wave packet as an assembly of test particles. However, the additional numerical expenditure precludes such an application at the present time. Reasonably stable nuclei and smooth phase space densities have been achieved with $L$ values of 1.5 and $2.1 \mathrm{fm}$. The dependence of the results on $L$ is studied below.

The propagation of those wave packets takes place via two- and three-body local Skyrme interactions, plus a long-range Coulomb potential. The Skyrme interaction can be written as

$$
V^{\mathrm{Sk}}=t_{0} \delta\left(\mathbf{r}_{i}-\mathbf{r}_{j}\right)+t_{3} \delta\left(\mathbf{r}_{i}-\mathbf{r}_{j}\right) \delta\left(\mathbf{r}_{i}-\mathbf{r}_{k}\right) .
$$

With the finite width Gaussian density distribution of the nucleons, this local interaction is effectively 
TABLE I. Parameters of Eq. (5) for the hard $(H)$ and the soft $(S)$ EOS.

\begin{tabular}{ccccc}
\hline \hline$K$ & $\alpha$ & $\beta$ & $\gamma$ & EOS \\
\hline $200 \mathrm{MeV}$ & $-356 \mathrm{MeV}$ & $303 \mathrm{MeV}$ & $\frac{7}{6}$ & $S$ \\
$380 \mathrm{MeV}$ & $-124 \mathrm{MeV}$ & $70.5 \mathrm{MeV}$ & 2 & $H$ \\
\hline
\end{tabular}

equivalent to a finite range interaction between point particles with range parameters of the order of $L$. The total interaction is given by

$$
V=V^{\mathrm{Sk}}+\boldsymbol{V}^{\mathrm{Coul}} \text {. }
$$

The microscopic Skyrmetype two- and three-body interaction of Eq. (2) can be identified with the density dependent potential determining the nuclear EOS in the case of infinite nuclear matter:

$$
U_{(\mathrm{loc})}=\alpha\left(\rho / \rho_{0}\right)+\beta\left(\rho / \rho_{0}\right)^{2} .
$$

Since we want to employ equations of state which are characterized by different compressibilities we generalize the three-body potential to

$$
U_{(\text {loc })}=\alpha\left(\rho / \rho_{0}\right)+\beta\left(\rho / \rho_{0}\right)^{\gamma},
$$

which gives us the freedom to adjust the compressibility constant $K$.

The parameters of Eq. (5) are adjusted to reproduce the ground-state properties of nuclear matter. Two parameters can be fixed if we determine the potential depth at the ground-state density $\rho_{0}$ with $E / A\left(\rho=\rho_{0}\right)=-16$ $\mathrm{MeV}$. The third parameter can be fixed by the choice of the compressibility constant $K$. The values used are $K=200 \mathrm{MeV}$ and $K=380 \mathrm{MeV}$, which simulate a soft or a hard EOS, respectively. The parameters for these interactions are given in Table $I$.

It has recently been emphasized ${ }^{33-35}$ that nonequilibrium effects can play an important role in a realistic treatment of heavy-ion collisions. First there is the momentum dependence of the nuclear interactions, which leads to an additional repulsion between the nucleons in heavy-ion collisions. It has been shown ${ }^{22}$ that the inclusion of such momentum dependent interactions (MDI) reduces the yield of pions and kaons substantially, while the transverse momentum transfer for $E \geq 400 A \mathrm{MeV}$ (Refs. 22 and 42) increases.

For the computation of the MDI we parametrized the real part of the optical potential in the following way: ${ }^{22}$

$$
U^{\mathrm{MDI}}=t_{4} \ln ^{2}\left[t_{5}\left(\mathbf{p}_{1}-\mathrm{p}_{2}\right)^{2}+1\right] \delta\left(\mathbf{r}_{1}-\mathbf{r}_{2}\right)
$$

with the parameters $\left(t_{4}=1.57 \mathrm{MeV}, t_{5}=5 \cdot 10^{-4}\right.$ $\mathrm{MeV}^{-2}$ ). The present expression for the MDI reproduces the experimental data up to energies $E \approx 1 A \mathrm{GeV}$. The parametrization of the real part of the optical poten-

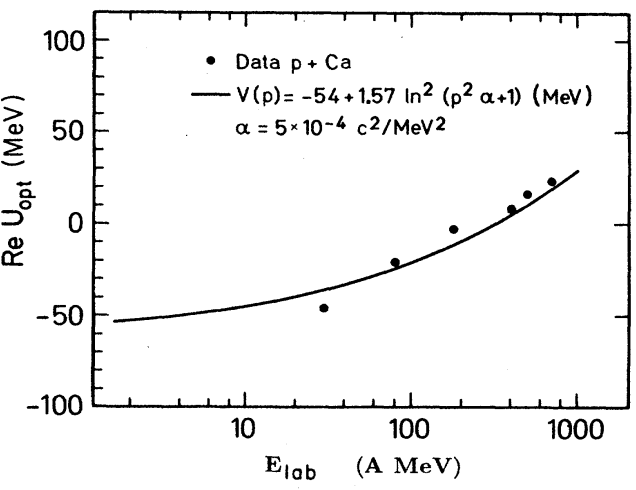

FIG. 1. Parametrization of the real part of the optical proton-nucleus potential: The solid circles indicate the data points, while the solid line is the fit to the data for the parametrization as indicated.

tial together with the data is shown in Fig. 1. It substitutes the term proportional $\Delta \mathrm{p}^{2}$ in the Skyrme interaction, which is in striking contrast with the data at $E_{\text {lab }} \geq 150 A \mathrm{MeV}$.

In order to reproduce the ground-state properties of nuclear matter with MDI one has to add this term into Eq. (5), which now reads for nuclear matter at rest

$$
U=\alpha\left(\rho / \rho_{0}\right)+\beta\left(\rho / \rho_{0}\right)^{\gamma}+\delta \ln ^{2}\left[\epsilon\left(\frac{\rho}{\rho_{0}}\right)^{2 / 3}+1\right] \rho / \rho_{0},
$$

where the average relative momenta are determined using the Thomas-Fermi approximation. The parameters have to be readjusted in order to obtain the same compressibilities in the ground state (see Table II). From now on we refer to the soft (hard) EOS plus MDI as SM (HM), respectively.

The resulting compressional energy is shown in Fig. 2 for the soft $(S)$ and the hard $(H)$ equation of state and for the soft and hard equation of state with MDI, (SM,HM). Note that all equations of state give the same ground state binding $\left(E / A=-16 \mathrm{MeV}\right.$ at $\left.\rho=\rho_{0}\right)$, but they differ drastically for higher densities. Here the hard EOS leads to much more compression energy than the soft EOS at the same density. The inclusion of the momentum dependent interactions leads for infinite nuclear matter at rest to almost no difference between the cases $S, \mathrm{SM}$ and $H, \mathrm{HM}$, respectively. This changes drastically if one considers heavy-ion collisions: The additional repulsion due to the separation of projectile and target in momentum space shifts the curve for the SM, (HM) interactions to higher energies. This can also be seen in the density, where we observe a decrease of the local density

TABLE II. Parameters of Eq. (7) for a hard and a soft local potential plus MDI.

\begin{tabular}{ccccccc}
\hline \hline$K$ & $\alpha$ & $\beta$ & $\gamma$ & $\delta$ & $\epsilon$ & EOS \\
\hline $200 \mathrm{MeV}$ & $-390 \mathrm{MeV}$ & $320 \mathrm{MeV}$ & 1.14 & $1.57 \mathrm{MeV}$ & $21.54 \mathrm{MeV}^{-2}$ & $\mathrm{SM}^{-2}$ \\
$380 \mathrm{MeV}$ & $-130 \mathrm{MeV}$ & $59 \mathrm{MeV}$ & 2.09 & $1.57 \mathrm{MeV}$ & $21.54 \mathrm{MeV}^{-2}$ & $\mathrm{HM}^{-2}$ \\
\hline \hline
\end{tabular}


in the central region of the reaction of half a unit when the MDI are added to the local potential. ${ }^{24}$

For the propagation of the centroids of the wave packets Hamilton's equations

$$
\begin{gathered}
\dot{r}_{i}=\frac{\partial H}{\partial p_{i}}, \\
\dot{p}_{i}=-\frac{\partial H}{\partial r_{i}}
\end{gathered}
$$

are integrated numerically using a second order integration routine with a fixed time step $\Delta t$.

Another in-medium effect with a large influence on the observables is the reduction of the nucleon-nucleon scattering cross section in the nuclear medium, due to the Pauli blocking of intermediate scattering states. ${ }^{65,66,43}$

It is well known that the $n-n$ cross section in infinite nuclear matter is lowered as compared to the free case. One part of this reduction results from the Pauli blocking of the final states. This reduction leads to a reduced cross section $\sigma_{N N}^{\mathrm{UU}}$, which is included in the collision term taken from the Uehling-Uhlenbeck transport equation which includes the Pauli blocking of the final states. ${ }^{15-17,38-44}$ In a Dirac-Brueckner theory the $n-n$ scattering also includes the Pauli blocking of intermediate states. This leads to an additional reduction of the $n-n$ cross section which can be approximated ${ }^{65}$ by a simple reduction factor

$$
\sigma_{N N}^{\mathrm{eff}} \approx 0.7 \cdot \sigma_{N N}^{\mathrm{UU}}
$$

The EOS itself is, of course, not influenced by the reduced cross sections. QMD calculations, which employ both the in-medium corrected cross sections and the MDI are denoted by the abbreviations SIM (HIM) depending on whether a soft or a hard local potential has been used.

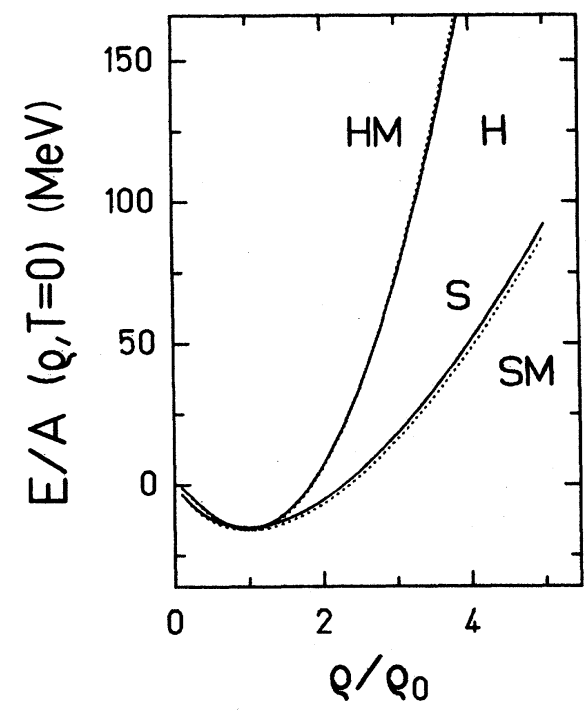

FIG. 2. The density dependence of the equation of state (compression energy per particle) for infinite cold nuclear matter is displayed for the different interactions. The solid line corresponds to the hard EOS $(H)$, the dashed line to the soft EOS $(S)$, while the dotted lines result from the soft $(\mathrm{SM})$ and hard (HM) local potential with additional momentum dependent interactions.
Two nucleons collide if their distance in configuration space is less than $r=\sqrt{\sigma / \pi}$. The scattering angle of a single nucleon-nucleon collision is chosen randomly in a way that the integral over the scattering angle agrees with the measured angular and energy distribution for elastic $p$ - $p$ scattering. ${ }^{36}$

In order to test, whether initialization of the projectile and target nucleus yields nuclei which exhibit the proper stability, ground state density and energy, we studied the stability of a single nucleus in a time interval which is characteristic for a heavy-ion collision. Randomly chosen coordinates of the wave packets do not create the real ground state, but excited states, which are unstable and evaporate particles within $t \leq 50 \mathrm{fm} / c$. We take into account the Pauli principle in the initial state by determining the positions of the nucleons randomly in a sphere in coordinate space with a radius $R=1.14 A^{-1 / 3}$, but requiring in addition a minimum distance $R_{\min }=1.5$ fm between any two nucleons. This yields a smooth density distribution in configuration space. The momenta of the nucleons are then randomly chosen in the interval $0 \leq|p| \leq p_{F}$, where the local Fermi momentum $p_{F}$ is determined from the local density via the Fermi gas approximation. A minimum distance $\Delta p=180 \mathrm{MeV} / c$ in momentum space is also required to fulfill the Pauli principle.

We are presently implementing an explicit Pauli potential, ${ }^{57}$ which ensures that also the equations of motion (8) respect the Pauli principle. Then the Hamiltonian has a true ground state with Fermi motion, which allows for a meaningful determination of the excitation energy of the produced complex fragments.

Figure 3 shows the time evolution of the root mean square (rms) radii of $\mathrm{Au}, \mathrm{Ag}, \mathrm{Ca}$, and $\mathrm{Ne}$ nuclei, averaged over 10 events, respectively. We have tested the stability with different time steps $\Delta t$ in the integration routine. The rms radii of the heavy nuclei exhibit oscillations about the mean values $(\Delta R / R \approx 10 \%)$ for all timesteps considered. That means that the nuclei are reasonably stable for time scales $t \leq 200 \mathrm{fm} / c$. The oscillations are due to the fact that our initial conditions do not correspond to the true quantum ground state, in which the fragmentation is expected to take place, since there is still some excitation energy in the system.

The same results are found for the neon nucleus for time steps $\Delta t \leq 0.4 \mathrm{fm} / c$. For $\Delta t=0.5 \mathrm{fm} / c$ we observe that the nuclei become instable after $t>100 \mathrm{fm} / c$. It should be noted that this instability of light nuclei is due to the initialization and is not observed in the light fragments after the collision, since the relative momenta of the constituents of a fragment are much smaller because otherwise the fragment would break up in the course of the fragmentation stage of a reaction. For the subsequent collision studies we choose $\Delta t=0.4 \mathrm{fm} / c$ at $E_{\text {lab }} \leq 200 A$ $\mathrm{MeV}$ and $0.2 \mathrm{fm} / c$ at $E_{\mathrm{lab}}>200 \mathrm{~A} \mathrm{MeV}$.

\section{THE MECHANISM OF FRAGMENT FORMATION AND MULTIFRAGMENTATION}

A. Time evolution of the fragmentation process

Let us first consider the reaction ${ }^{197} \mathrm{Au}+{ }^{197} \mathrm{Au}$ at an energy of $200 \mathrm{~A} \mathrm{MeV} .200$ simulations have been carried 


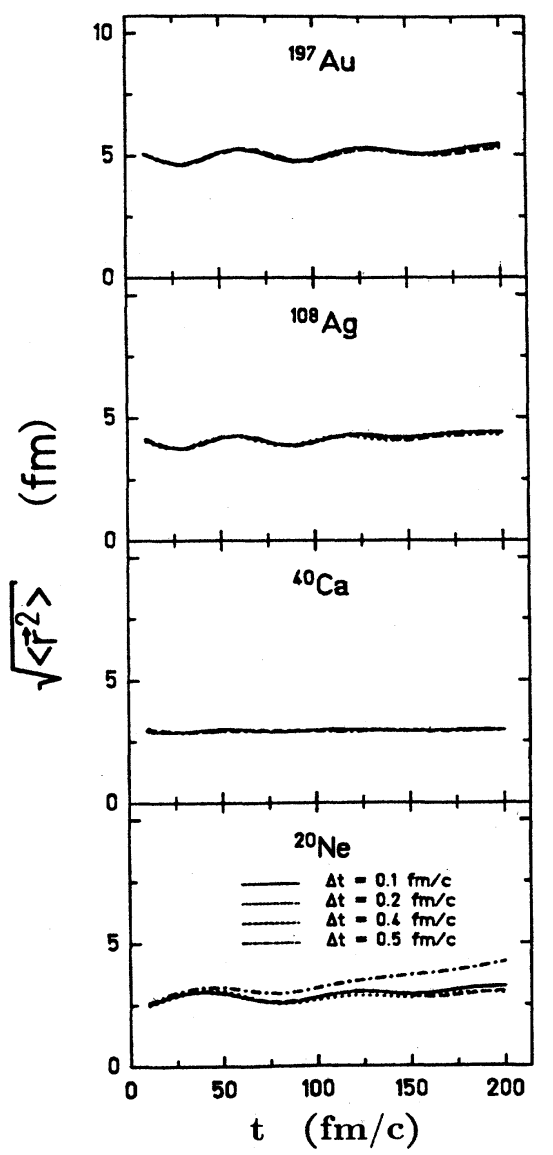

FIG. 3. Stability of the nuclei: The time evolution of the root mean square radii for the nuclei $\mathrm{Au}, \mathrm{Ag}, \mathrm{Ca}$, and $\mathrm{Ne}$ are depicted for different time steps $\Delta t$ in the integration routine.

out for impact parameters $1,3,5$, and $7 \mathrm{fm}$ and for a hard $(H)$ and a soft $(S)$ EOS. We follow the evolution of the reaction for $200 \mathrm{fm} / c$. This time is long enough to study the final fragment distribution, since as we will see later, the mass yields reach their asymptotic values after approximately $150 \mathrm{fm} / c$ reaction time. In order to study fragment formation we recorded the position and momenta of each nucleon every $10 \mathrm{fm} / c$. Then we determined the cluster distribution, using a common minimum spanning tree procedure. ${ }^{38}$

Two nucleons are considered to be bound in a fragment if the centroids of their wave packets have a spatial distance $d_{0} \leq 3 \mathrm{fm}$. We checked that the results do not depend sensitively on the correlation length $d_{0}$, since nucleons which belong to different clusters are widely spread in configuration space after $200 \mathrm{fm} / c$. Doubling of $d_{0}$ leads to a $\approx 20 \%$ shift of the yields of the complex fragments. Because of the strong correlation between position and momentum of the nucleons forming a fragment, it is not necessary to consider the relative momenta of the nucleons forming a cluster at late times.

We have grouped the fragments into seven mass intervals. The first interval contains all free nucleons $(A=1)$, the second light fragments with $A=2-4$, then follow the mass ranges with $A=5-15,16-30,31-50,51-70$, and

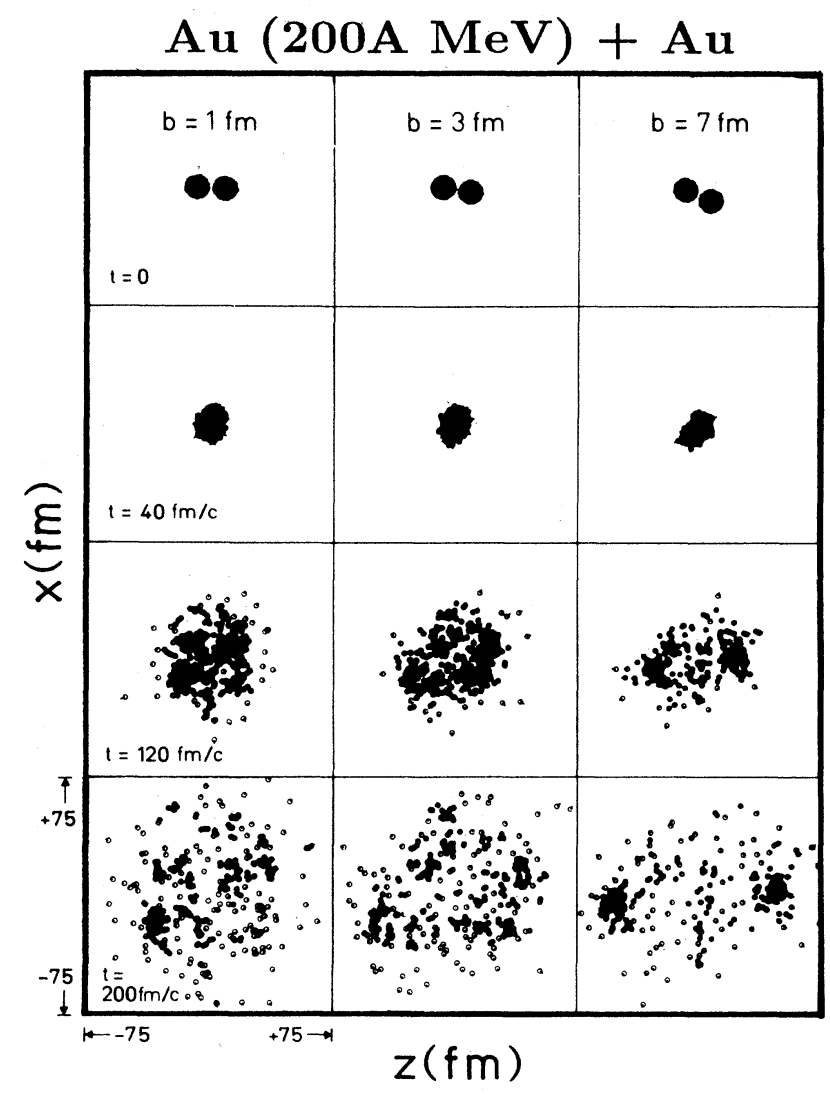

FIG. 4. Time evolution of the particle distribution in configuration space for the reaction $\mathrm{Au}(200 \mathrm{~A} \mathrm{MeV})+\mathrm{Au}$ for impact parameters of $b=1,3$, and $7 \mathrm{fm}$. The projection of all particles in the reaction plane $(x-z)$ is displayed for four different times as indicated.

\section{$A>70$.}

The spatial evolution of a $\mathrm{Au}+\mathrm{Au}$ collision at $200 \mathrm{~A}$ $\mathrm{MeV}$ energy can be followed in Fig. 4 for impact parameters 1,3 , and $7 \mathrm{fm}$. The beam axis coincides with the $z$ direction. Observe the formation of one blob of matter for all impact parameters $(t \leq 40 \mathrm{fm} / c)$, which then disintegrates and yields the fragments. For half overlap collisions $(b=7 \mathrm{fm})$ two massive projectile and target remnants survive the reaction. In this case one observes almost no intermediate mass fragments. When going to more central collisions the projectile and targetlike fragments become smaller $(b=3 \mathrm{fm})$ and are absent in the most central collisions, where we observe a complete disintegration of the incident nuclei. On the other hand the intermediate mass fragments are abundantly produced in central collisions and disappear for peripheral ones.

In Fig. 5 we show the typical time structure of a single half overlap collision for $\mathrm{Au}(200 A \mathrm{MeV}, b=7 \mathrm{fm})+\mathrm{Au}$ in more detail. Only fragments with $A \geq 10$ are considered from $t=0$ (bottom) to $t=200 \mathrm{fm} / c$ (top) in steps of $10 \mathrm{fm} / c$. Up to $50 \mathrm{fm} / c$ one observes one blob of matter in configuration space which - for this large impact parameter-is still separated in momentum space into a projectile and a targetlike residue [for central col- 


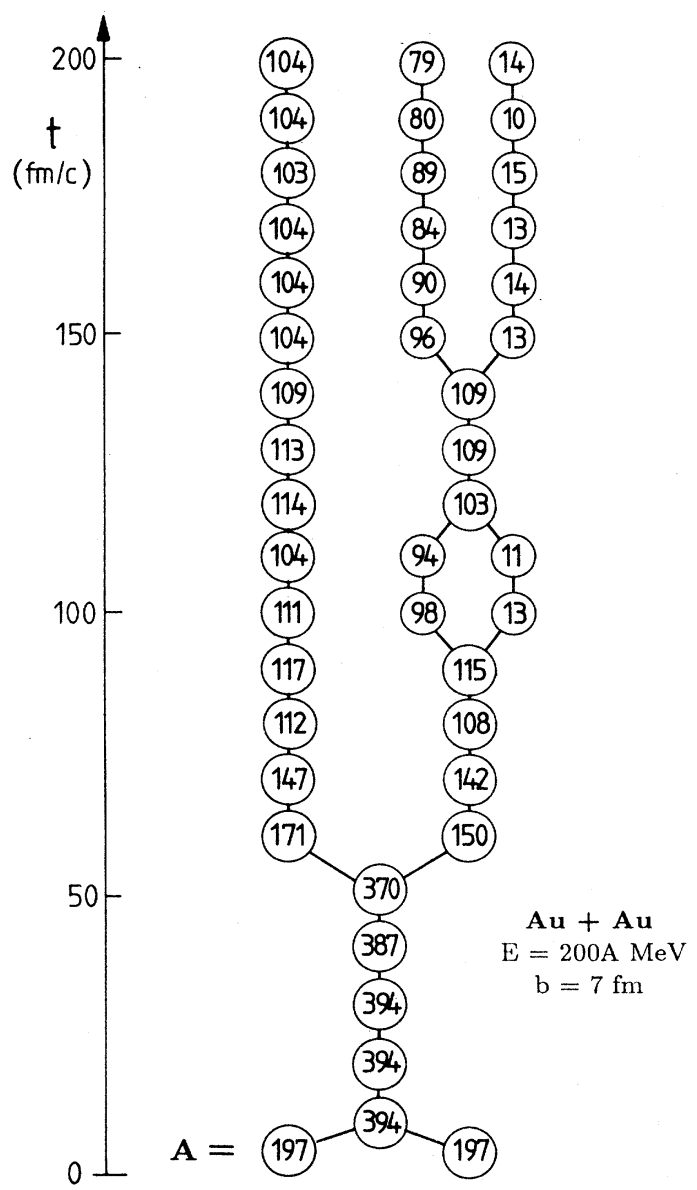

FIG. 5. Time evolution of the fragmentation process in a single event for the reaction $\mathrm{Au}(200 A \mathrm{MeV}, b=7 \mathrm{fm})+\mathrm{Au}$. Shown are all massive fragments $(A \geq 10)$ from $t=0 \mathrm{fm} / c$ (bottom) to $t=200 \mathrm{fm} / c$ (top). Two nucleons are considered to be members of a fragment if their spatial distance is less than $d_{0}=3 \mathrm{fm}$.

lisions ( $b \leq 3 \mathrm{fm}$ ), this is not the case; we then observe complete stopping of projectile and target in the $\mathrm{cm}$ frame]. After $50 \mathrm{fm} / c$ the system breaks up into these two residues. Between 30 and $80 \mathrm{fm} / c$ most of the single nucleons and light fragments $(A<10)$ are emitted. Then a rather stable fragment with $A \approx 105$ remains in the projectile rapidity regime. In the target regime a second breakup is observed, which yields two stable fragments with $A \approx 80$ and 15 , respectively. At large impact parameters $(b=7 \mathrm{fm})$ the intermediate mass fragments are mostly produced in the binary breakup of the heavy residues. A similar conclusion has recently been reached experimentally for asymmetric systems. ${ }^{67}$

Figure 6 presents the average number of fragments as a function of time for $b=3 \mathrm{fm}$. First of all we observe that the mass yield distribution for all fragments $(A \geq 2)$ stabilizes at $150 \mathrm{fm} / c$ or even earlier.

Let us first concentrate on the heavy clusters with $A>70$. Here one recognizes one cluster up to $50 \mathrm{fm} / c$ which is not stable and decays rapidly. The decay chain can be seen by the subsequent population and depopula-

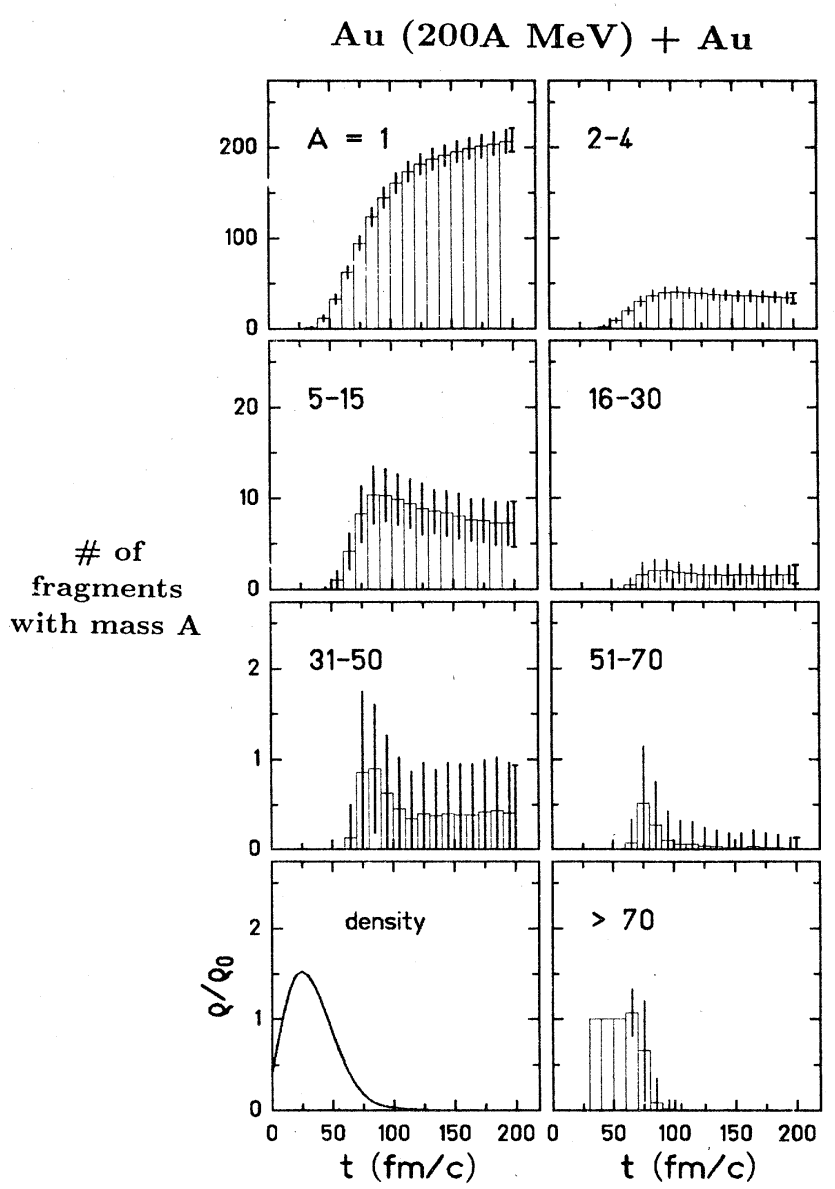

FIG. 6. Time dependence of the fragment yields for the reaction $\mathrm{Au}(200 A \mathrm{MeV}, b=3 \mathrm{fm})+\mathrm{Au}$, based on 200 collisions. The average number of fragments for different mass classes as indicated are shown as a function of reaction time. The width of the fragment distributions are indicated by the error bars. Also shown is the time evolution of the local density in the central region of the reaction. The local density has been determined in a sphere with the radius $r=2 \mathrm{fm}$ in the center of the c.m. frame.

tion of the different mass bins for the smaller clusters. Along the decay chain the cluster emits also single nucleons and therefore these numbers increase but have almost saturated at $t=200 \mathrm{fm} / c$.

The clusters in between $2 \leq A \leq 30$ have a completely different history. They are formed at a very early stage of the reaction and do not get fed from the decaying remnants nor do they decay. After $100 \mathrm{fm} / c$ practically all of them have been formed. They emerge from the surface region of the combined system ${ }^{26}$ and measure the violence of the reaction.

Keep in mind that the transient appearance of large "clusters" reflects the simple configuration space method used to define the clusters. The actual phase space distributions indicate that the cluster correlations are established much earlier. This can be seen in the transverse momentum transfer discussed below. The complex fragments are most sensitive to the detailed dynamics during 
the early compression stage. The transverse momentum transfer, which is built up during the expansion from the high density stage can be seen most clearly for the intermediate mass fragments. This indicates that these fragments have been formed early as prefragments in the shock zone and therefore show this strongly enhanced sensitivity on the equation of state.

Also shown in Fig. 6 is the time evolution of the local density in the central region of the reaction. Observe the compression shock at $t \approx 20 \mathrm{fm} / c$. At that time also the highest temperatures are found. Subsequently the system rapidly expands out of the central region of the shocked matter. Note that the highest compression occurs at a time when in configuration space fragments are not yet separated. After the central density has decreased they are visible as individual entities only.

We have checked the dependence of the calculated fragment yields on the width of the Gaussians (8), i.e., the effective range of the interaction. While the light fragment yields ( $A=1-4$ ) change only by $10 \%$, the number of massive fragments decreases by $30 \%$ when $L^{2}$ is doubled. We hope to remedy this dependence on $L$ by including an explicit long range Yukawa interaction in the near future.

\section{B. Mass distributions and the liquid-vapor phase-transition}

Figure 7 shows the inclusive, i.e., impact parameter averaged mass yields for the hard and the soft equation of state for $\mathrm{Au}(200 \mathrm{~A} \mathrm{MeV})+\mathrm{Au}$. Both curves exhibit a clear power law behavior $Y(A) \sim A^{-\tau}$. The fragment yields, however, are not sensitive to the underlying EOS. For the constant $\tau$ we find $\tau \approx 2.3$. The same behavior is found in the asymmetric system $\mathrm{Ne}+\mathrm{Au}$ at $1 A \mathrm{GeV}$ bombarding energy. ${ }^{53,26} \mathrm{We}$ want to emphasize that a change of the correlation length $d_{0}$ in the minimum spanning tree procedure from $d_{0}=3 \mathrm{fm}$ to $d_{0}=4 \mathrm{fm}$ does not change this power law dependence of $Y(A)$. Such a power-law dependence with an exponent $2 \leq \tau \leq 3$ has been interpreted as an evidence for a liquid-vapor phase transition. ${ }^{45-49}$ In the present fully dynamic model we can investigate to what extent this conclusion is conclusive.

Figure 8 displays the dependence of the final fragment yields on the impact parameter. In all cases we observe a

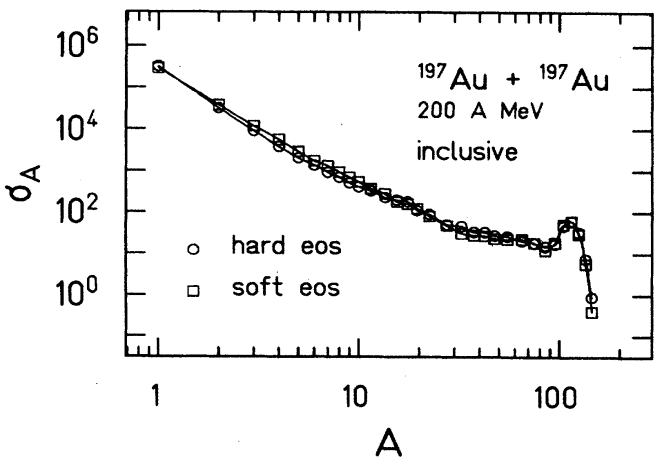

FIG. 7. Inclusive, i.e., impact parameter averaged mass yield for the reaction $\mathrm{Au}(200 \mathrm{~A} \mathrm{MeV})+\mathrm{Au}$ obtained with the soft (squares) and the hard (circles) EOS, respectively.

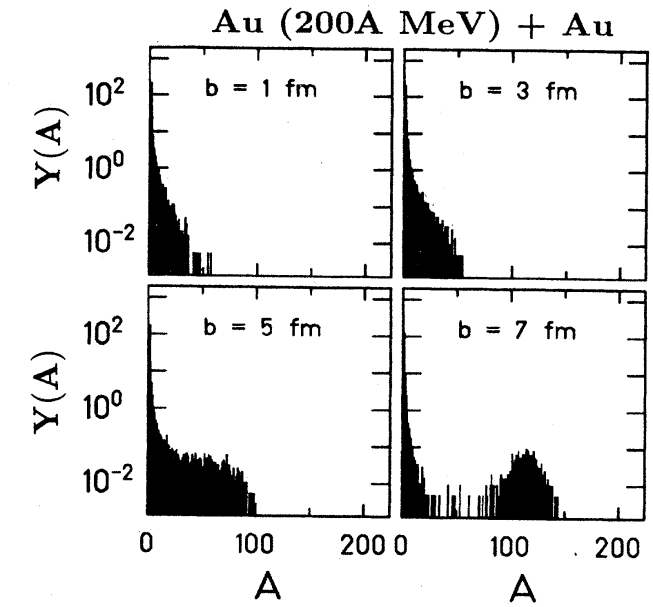

FIG. 8. Impact parameter dependence of the average mass yield per event $Y(A)$ for the $\mathrm{Au}(200 A \mathrm{MeV})+\mathrm{Au}$ reaction.

steep drop of the yields of fragments with $A \leq 10$. Large differences become evident for the heavier fragments. For central collisions ( $b=1 \mathrm{fm}$ ) the projectile and target are completely disintegrated, and hence there are no $A>40$ fragments. For $b=5 \mathrm{fm}$ the distribution exhibits a flat plateau between $A=40-70$. At $b=7 \mathrm{fm}$ a $U$ shaped curve with a peak at $A \approx 120$ (projectile and target residue) and almost no fragments in the $A=20-80$ region results. This systematic has already been discussed in Fig. 4.

Hence, we conclude that impact parameter averaging (rather than a liquid-vapor phase transition) leads to an accidental power law dependence of the inclusive mass yield.

Furthermore, with respect to the liquid-vapor phase transitions, we find that for noncentral collisions the fragments of different masses reside in different rapidity bins (see also Fig. 18) in each event. However, for very central ( $b<2 \mathrm{fm}$ ) collisions, where we do observe a complete stopping and total disintegration of the projectile and the target, all fragments do reside in the same rapidity interval. Therefore we propose to study the occurrence of the liquid-vapor phase transition by measuring the fragment yield excitation function for intermediate energies ( $E=30-200 A \mathrm{MeV}$ ). One should trigger on central collisions of very heavy projectiles, which provide sufficient stopping power. This excitation function is shown in Fig. 17 (see text following).

Let us now briefly discuss the dependence of the final fragment yield $Y(A)$ on bombarding energy and the EOS. The excitation function of the fragment distributions has been calculated for the $\mathrm{Au}+\mathrm{Au}$ collisions up to $800 \mathrm{~A} \mathrm{MeV}$ bombarding energy. However, up to now the only data available are for $E_{\text {lab }}=200 A \mathrm{MeV}$. Because of the computational expenditure for the $\mathrm{Au}+\mathrm{Au}$ system only 50 events per $E_{\text {lab }}$ have been studied at one impact parameter ( $b=3 \mathrm{fm}$ ).

Figure 9 shows the final fragment yields per event (after $t=100 \mathrm{fm} / c)$ for $\mathrm{Au}(b=3 \mathrm{fm})+\mathrm{Au}$ collisions at 200,400 , and $800 A \mathrm{MeV}$ beam energy for the case of the 


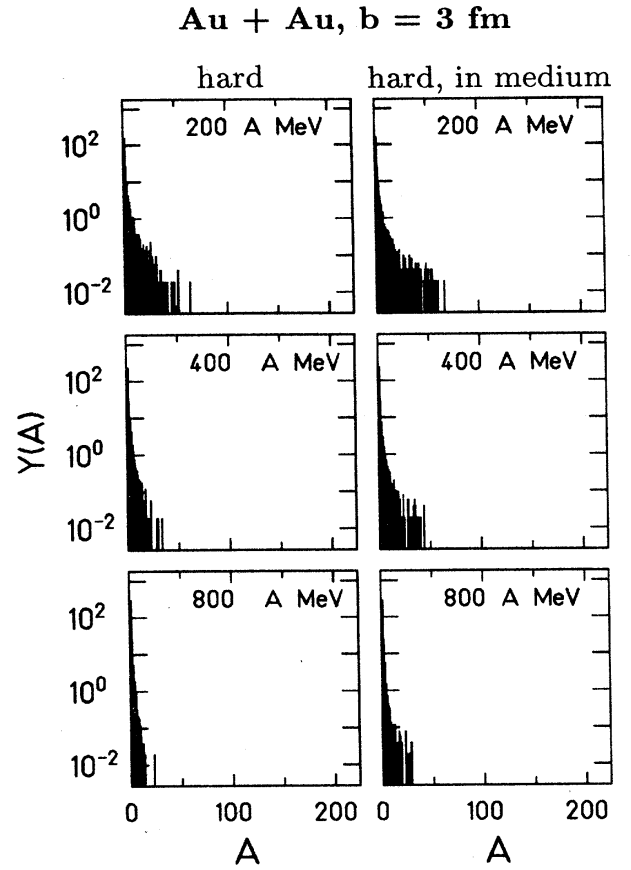

FIG. 9. Energy dependence of the average mass yield per event $Y(A)$ for the reaction $\mathrm{Au}(b=3 \mathrm{fm})+\mathrm{Au}$. The left column shows the results obtained with the hard local potential alone $(H)$, while the right column shows the yields obtained with the hard local potential plus additional medium corrections (HIM), i.e., reduced $N$ - $N$ scattering cross sections and momentum dependent interactions.

hard local potential without in-medium effects (left column, $H$ ) and with the in-medium effects included (right column, HIM). Note that the fragment yields are sensitive to the medium corrections. The in-medium reduced cross sections lead at all energies to the formation of more heavy fragments. We want to point out that the reduction of the cross sections is associated with less stopping, i.e., larger transparency, and therefore with less thermalization and disintegration of the system (see also Sec. IV and Ref. 24). Therefore more massive fragments survive the reaction. This effect might be useful to determine the in-medium cross section experimentally.

Independent of the form of the local potential there is a rapid decrease of the fragment yields for the light fragments $(A<10)$ at all energies. The slope of $Y(A)$ becomes steeper with increasing bombarding energy in agreement with simple thermostatic predictions, which assume that the entropy of the system increases with bombarding energy. ${ }^{51,52}$ At $E \geq 400 A \mathrm{MeV}$ and $b=3 \mathrm{fm}$ there are almost no fragments with $A>20$ left. In this case only the light fragment yield exceeds one fragment per event.

\section{Light fragment production and total multiplicities}

We now proceed to study the light fragment emission in more detail. The corresponding data for $\mathrm{Nb}+\mathrm{Nb}$ have recently been published. ${ }^{70}$

One reason why we are interested in the light particle yields is their connection to the entropy in heavy ion reactions. It has been proposed ${ }^{68}$ to determine the entropy in heavy ion collisions via the deuteron to proton yields $\boldsymbol{R}_{d p}$ using the following formula

$$
\frac{S}{A}=3.945-\ln R_{d p} \text {. }
$$

Light fragments heavier than the deuteron can be included in the determination of the entropy by replacing the $\boldsymbol{R}_{d p}$ ratio of Eq. (10) by the ratio of the deuteronlike to the protonlike fragments $\widetilde{R}_{d p},{ }^{69}$ with

$$
\widetilde{R}_{d p}=\frac{d+\frac{3}{2}\left(t+{ }^{3} \mathrm{He}\right)+3{ }^{4} \mathrm{He}}{p+d+t+2\left({ }^{3} \mathrm{He}+{ }^{4} \mathrm{He}\right)} .
$$

However, it was shown ${ }^{51,52}$ that stable and unstable isotopes lead to a more complex nonanalytic relation between the $d_{\text {like }} / p_{\text {like }}$ ratios and the entropy, which is approximately independent of the breakup density.

Here we present the impact parameter and bombarding energy dependence of the ratios of the light fragments to protons $(X / p)$ where $X$ stands for fragments with $A=2$, 3 , and 4 . They were also determined by the minimum spanning tree procedure and not by the quantum scattering description of Ref. 22. Since there is no isospin identification in the QMD model we cannot distinguish between different isobars, i.e., the $A=3$ fragments represent both $t$ and ${ }^{3} \mathrm{He}$. The calculated (global) ratios include the whole phase space, while the data give the (local) light particle ratios and the (local) entropy only for a limited region of phase space where the plastic ball acceptance of $d_{\text {likes }}$ and $p_{\text {likes }}$ overlap. ${ }^{70}$ For these reasons we cannot present a one to one comparison between theory and experiment.

In order to compare the general behaviors, i.e., multiplicity and bombarding energy dependence of $\widetilde{R}_{d p}$ we assume the $d_{\text {like }} / p_{\text {like }}$ ratios in the QMD model to be

$$
\widetilde{R}_{d p}=\frac{Y(A=2)+\frac{3}{2} Y(A=3)+3 Y(A=4)}{N_{p}},
$$

where $Y(A=n)$ stands for the number of fragments with mass $n$ in one event. In analogy to the experimental results we define the total participant proton multiplicity $N_{p}$ in the following way:

$$
\begin{aligned}
N_{p}=\frac{Z_{p}+Z_{T}}{A_{P}+A_{T}}[ & Y(A=1)+2 Y(A=2) \\
& +3 Y(A=3)+4 Y(A=4)] .
\end{aligned}
$$

These $N_{p}$ values now can be compared to the data, which include all participants and not only those in the overlap-phase-space window.

Let us first study the impact parameter dependence of the participant proton multiplicities $N_{p}$, defined in Eq. (13). This relation is shown in Fig. 10 for the reaction $\mathrm{Nb}+\mathrm{Nb}$ at $250 A \mathrm{MeV}$ beam energy. A nearly linear increase of $N_{p}$, when going from $b=7 \mathrm{fm}$ to $b=2 \mathrm{fm}$ is observed. However, there is no significant increase of the participant proton multiplicity when going from $b=2 \mathrm{fm}$ to $b=0 \mathrm{fm}$. From this one can conclude that all collisions up to a scaled impact parameter $\widetilde{b}=b /\left(R_{1}+R_{2}\right)$ 


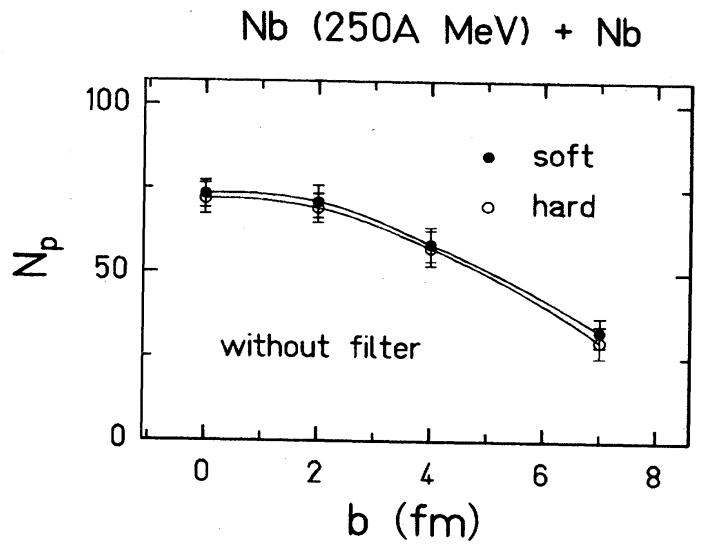

FIG. 10. The relation between the impact parameter $(b)$ and the average multiplicity of participant protons $\left(N_{p}\right)$ for the reaction $\mathrm{Nb}(250 A \mathrm{MeV})+\mathrm{Nb}$ is shown for the hard and the soft EOS as indicated. The error bars indicate the width of the $N_{p}$ distributions.

$\approx 0.2$ are experimentally considered as central collisions and cannot be distinguished, if one chooses the participant proton multiplicity $N_{p}$ as a measure of $b . N_{p}$ can be used to measure $b$ for intermediate and half overlap collisions only. This figure also shows that the EOS has almost no influence on $N_{p}$.

In Fig. 11 we present the calculated global $X / p$ ratios for the reaction $\mathrm{Nb}+\mathrm{Nb}$ for $250 A \mathrm{MeV}$ beam energy vs $N_{p}$. Here $X$ stands for the $A=2,3$, and 4 fragments. All curves exhibit the same general behavior: The abundance

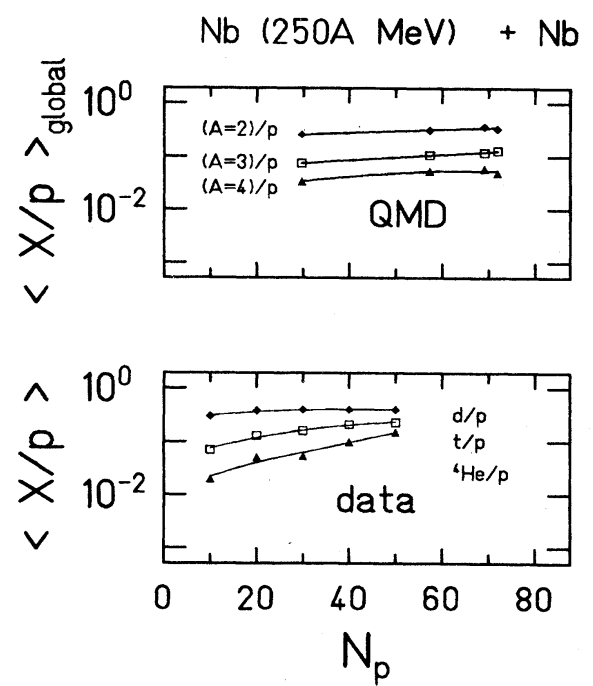

FIG. 11. The $X / p$ ratios for the reaction $\mathrm{Nb}(250 A$ $\mathrm{MeV})+\mathrm{Nb}$ as a function of $N_{p}$ are compared to the experimental data (taken from Ref. 70). The QMD results (upper figure) show the ratio of the average number of light fragments $(A=2,3,4)$ to the average number of free protons. The (global) yields have been obtained with the minimum spanning tree procedure and not by a quantum scattering procedure. The calculation takes into account the whole phase space, while the measured (local) $d / p, t / p$ and ${ }^{4} \mathrm{He} / p$ (lower figure) ratios were registered only in a limited phase space region where the plastic ball acceptance of these particles overlap.

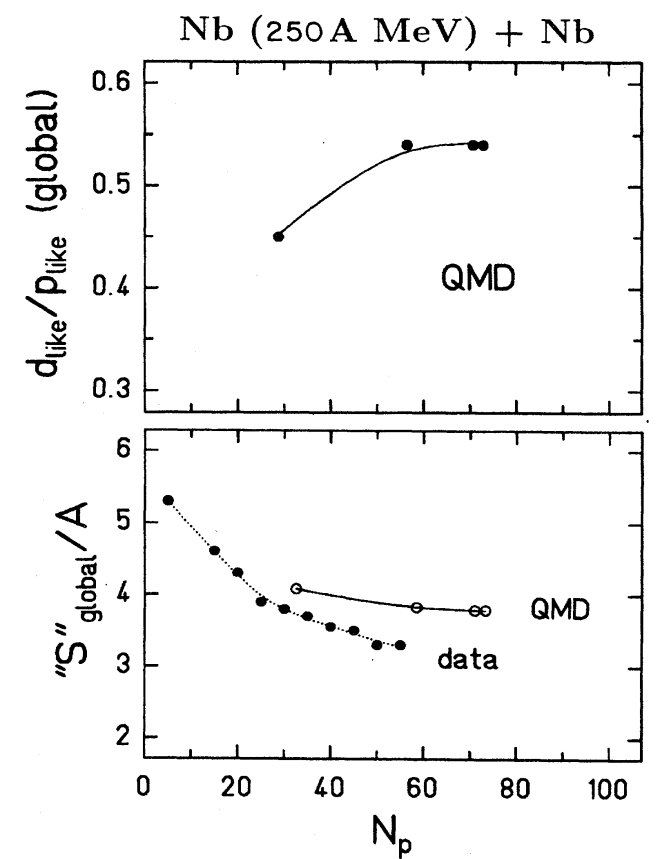

FIG. 12. Calculated $d_{\text {like }} / p_{\text {like }}$ ratio (upper figure) as a function of the participant proton multiplicity $N_{p}$ for the reaction $\mathrm{Nb}(250 A \mathrm{MeV})+\mathrm{Nb}$. The lower figure compares the measured to the calculated "entropy" per baryon. The "entropies" have been obtained from the $d_{\text {like }} / p_{\text {like }}$ ratios via the quantum statistical model (Ref. 52).

ratios of the light fragments increase with increasing $N_{p}$ (decreasing impact parameter) and reach an asymptotic value for central collisions. Again there is almost no difference between collisions with 0 or $2 \mathrm{fm}$ impact parameter. Also shown in this figure are the plastic ball data. ${ }^{70}$ The data curves are shown for the ratios of the $d$, $t$, and ${ }^{4} \mathrm{He}$ to protons, marked with the same symbols as the calculated $A=2 / p, 3 / p$, and $4 / p$ ratios. The general behavior of the light particle ratios agree qualitatively with the data. Our results are-within the admittedly large statistical uncertainties - independent of the Gaussian width, i.e., of the effective range of the potential, as well as of the equation of state.

Figure 12 (upper part) shows the global $d_{\text {like }} / p_{\text {like }}$ ratios defined in Eq. (12) vs $N_{p}$ for $250 \mathrm{~A} \mathrm{MeV}$ bombarding energy. Here one clearly observes that the probability for the production of light clusters increases with increasing proton multiplicity. It reaches a plateau for higher $N_{p}$ values.

Unfortunately the measured $d_{\text {like }} / p_{\text {like }}$ ratios are not published; the experimentalists give only ${ }^{70}$ "entropies" which are obtained from $\widetilde{R}_{d p}$ via the quantum statistical model (QSM). ${ }^{52}$ In order to compare to the data we used the same prescription to relate our $\widetilde{R}_{d p}$ to "entropies" and show in the lower part of Fig. 12 the multiplicity (impact parameter) dependence of the measured ${ }^{70}$ and calculated "entropies." For all energies the "entropy" decreases with increasing $N_{p}$ (decreasing impact parameter). We would like to emphasize here that we have not calculated the entropy in the QMD model, but merely ap- 


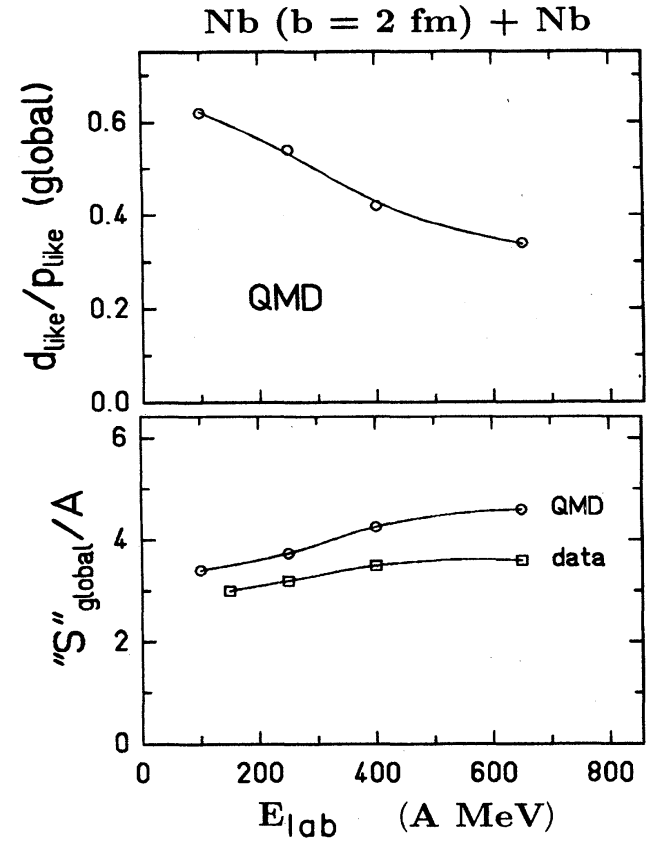

FIG. 13. Same as Fig. 12 but as a function of the bombarding energy (for central collisions).

plied the same simple QSM analysis ${ }^{51,52}$ which has been used by the experimentalists. Again a qualitative agreement with the data is observed.

Figure 13 shows the corresponding bombarding energy dependence of the $d_{\text {like }} / p_{\text {like }}$ ratios and of the measured and calculated "entropies." In agreement with the data we find an increase of the "entropy" with increasing bombarding energy.

\section{Multiplicity distribution of intermediate mass fragments}

Let us now come back to the $\mathrm{Au}+\mathrm{Au}$ system and study the behavior of the intermediate mass fragments in more detail. Here we want to compare our results directly with the plastic ball data. ${ }^{18,19}$ Therefore we applied the experimental efficiency filters; i.e., the same lowenergy cutoff at $35 \mathrm{MeV} /$ nucleon to all particles, the intermediate mass fragments are only counted if they were emitted with an angle less than $30 \mathrm{deg}$ in the laboratory system, corresponding to the experimental plastic mall setup, which has detected such intermediate mass fragments due to higher gains than the rest of the $4 \pi$ spectrometer. Fragments with masses greater than 20 $(Z \geq 10)$ are ignored. ${ }^{18,19}$

The multiplicity distribution $\left[M_{c}(A>4)\right]$ of the intermediate mass fragments, i.e., fragments with $A>4$ obtained with the large width $(L=2.1 \mathrm{fm})$ is shown in Fig. 14 for four different impact parameters. However, we do observe a $30 \%$ reduction in $\boldsymbol{M}_{c}$ when going from the small Gaussian width to the large value as noted in the end of Sec. III A. In the upper part we show the unfiltered results and in the lower part those ones obtained with this simple plastic ball filter. In the case of the unfiltered distributions we find for peripheral collisions typically $2-4$ heavy projectile and targetlike rem-

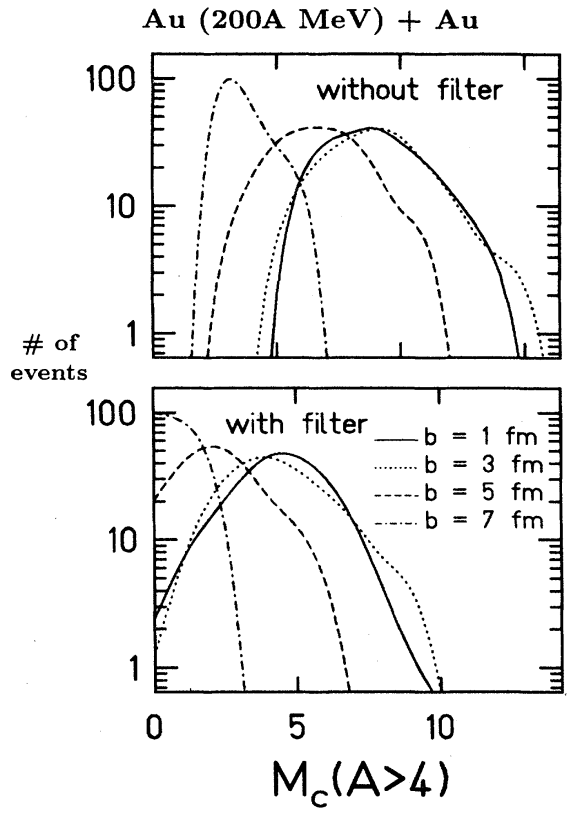

FIG. 14. Calculated multiplicity distributions of fragments with $A>4$ for the reaction $\mathrm{Au}(200 A \mathrm{MeV})+\mathrm{Au}$ at impact parameters of $b=1,3,5$, and $7 \mathrm{fm}$. The upper part shows the unfiltered yields, while the lower figure has been obtained using a plastic ball filter. The plastic ball filter rejects all particles and fragments with $E_{\text {lab }}<25 A \mathrm{MeV}$. The massive fragments (up to mass 20 ) are only registered if they are emitted with an angle less than $30^{\circ}$ in the laboratory frame. Fragments with masses greater than $20(Z \geq 10)$ are ignored.

nants. The number of intermediate mass fragments increases to a mean multiplicity of about 10 fragments per event for near central collisions ( $b=3$ and $1 \mathrm{fm}$ ).

The multiplicity distributions $M_{c}(5 \leq A \leq 20)$ of the remaining detectable fragments (lower part of Fig. 14) also shows that the near central collisions $(b=1,3 \mathrm{fm}$ ) lead to almost identical distributions. Both curves are now peaked at much smaller mean values of $\left\langle M_{c}\right.$ $(5 \leq A \leq 20)\rangle \approx 5$. Observe that in the half overlap reaction $(b=7 \mathrm{fm})$ the maximum of the distribution $M_{c}$ ( $5 \leq A \leq 20$ ) is at zero, i.e., the $2-4$ fragments at $b=7 \mathrm{fm}$ shown the upper part have escaped the detector window of the plastic ball, i.e., the heavy fragments from the target and projectile hemisphere are strongly suppressed. We also find that $M_{c}(A>4)$ is nearly independent of the EOS. $^{25}$

In Fig. 15 we compare the filtered multiplicity distributions of central and peripheral collisions $(b=3,10 \mathrm{fm}$ ) directly with the corresponding experimental data curves from Refs. 18 and 19 . The data are shown for those events with maximal (MUL4) and minimal (MUL1) participant proton multiplicities $N_{p}$. The data and theory are shown for fragments with $A>5$. Note the dependence of $M_{c}$ on the width $L$, i.e., the range of the interaction, when the same equation of state is used. This is due to the different binding energies, which in finite systems depend on the range of the interaction.

In order to relate the experimental multiplicity bins with our impact parameter we want to show the depen- 


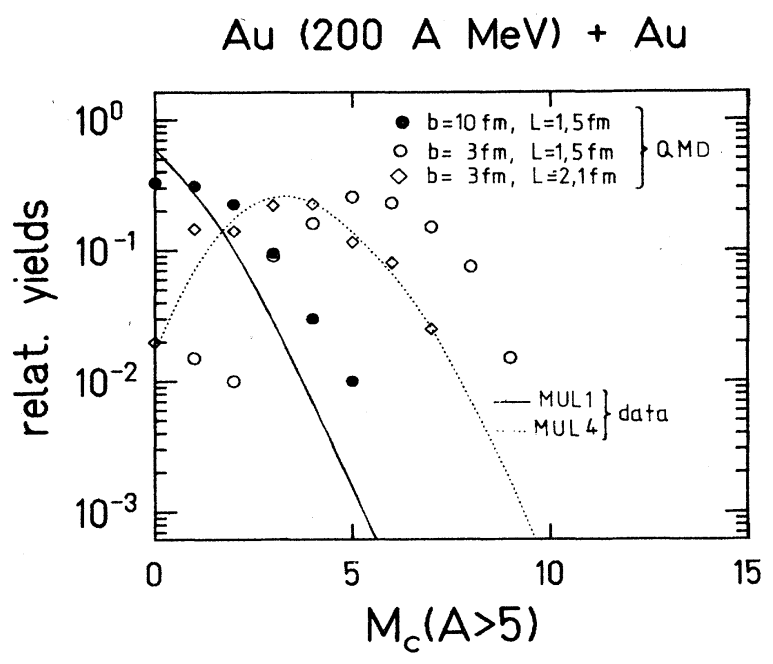

FIG. 15. Calculated vs measured fragment multiplicity distributions for peripheral (MUL1, $b=10 \mathrm{fm}$ ) and central (MUL4, $b=3 \mathrm{fm})$ collisions of the system Au $(200 A \mathrm{MeV})+\mathrm{Au}$. The QMD results (circles) have been obtained with the filter, while the lines represent the experimental data, taken from Refs. 18 and 19. The results obtained at $b=3 \mathrm{fm}$ with $L=2.1$ and 1.5 fm are shown.

dence of the calculated (filtered) $N_{p}$ distributions on the impact parameter. This relation is shown in Fig. 16 for the reaction $\mathrm{Au}(200 \mathrm{~A} \mathrm{MeV})+\mathrm{Au}$. Also shown in this figure are the experimental cuts (horizontal dashed lines). The multiplicity cuts given in Ref. 19 are labeled with MUL1 -.. MUL5 corresponding to the multiplicity intervals $0 \leq N_{p}<23$ (MUL1), $23 \leq N_{p}<46 \quad$ (MUL2), $46 \leq N_{p}<69$ (MUL3), $69 \leq N_{p}<92$ (MUL4), and $N_{p}>92$ (MUL5).

Our calculations show that impact parameters from 1 to $10 \mathrm{fm}$ cover the multiplicity distributions in the region $20 \leq N_{p} \leq 100$. The distributions are very narrow, there is no overlap in the multiplicity distributions. (Compare, however, Fig. 10, which shows the same relation for the $\mathrm{Nb}+\mathrm{Nb}$ system, without applying any filters. In that

\section{$\mathrm{Au}(200 \mathrm{~A} \mathrm{MeV})+\mathrm{Au}$}

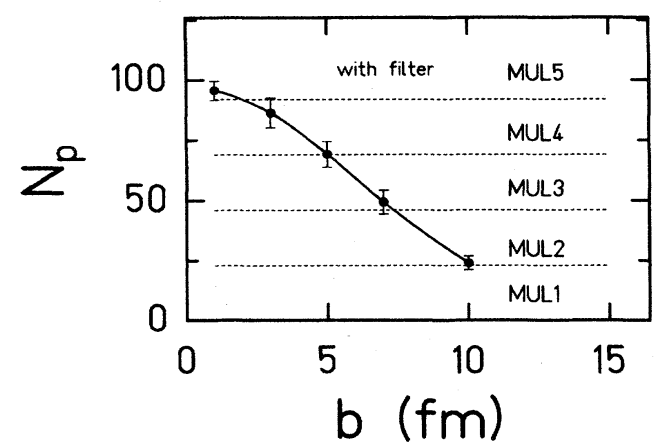

FIG. 16. Impact parameter dependence of the filtered average participant proton multiplicities $N_{p}$ for the reaction $\mathrm{Au}$ $(200 \mathrm{~A} \mathrm{MeV})+\mathrm{Au}$. The horizontal dashed lines depict the experimental multiplicity cuts (MUL1-MUL5). The bars indicate the statistical width of the distributions.

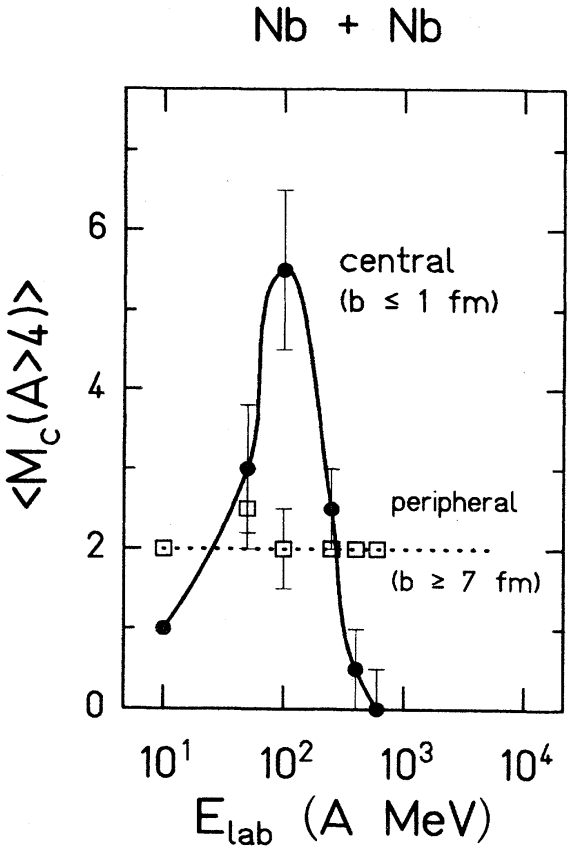

FIG. 17. Excitation function of the average multiplicity of intermediate mass fragments $M_{c}(A>4)$ for the system $\mathrm{Nb}+\mathrm{Nb}$ obtained without filters for central and peripheral collisions as indicated. The curves are to guide the eye only.

system we find no difference in $N_{p}$ for the most central collisions.) The mean values of this distribution are $\left\langle N_{p}\right\rangle \approx 25(b=10 \mathrm{fm}),\left\langle N_{p}\right\rangle \approx 50(b=7 \mathrm{fm}),\left\langle N_{p}\right\rangle \approx 70$ $(b=5 \mathrm{fm}),\left\langle N_{p}\right\rangle \approx 85(b=3 \mathrm{fm})$, and $\left\langle N_{p}\right\rangle \approx 95(b=1$ $\mathrm{fm})$. With these results at hand we have connected the experimental multiplicity bins with the impact parameter: We find that apparently the bin MUL1 results from collisions with impact parameters $b \geq 10 \mathrm{fm}$. The MUL2 bin corresponds approximately to collisions with 7 $\mathrm{fm} \leq b \leq 10 \mathrm{fm}$, while the impact parameters $b=5$ and 3 fm roughly represent the MUL3 and MUL4 bins. The highest experimental multiplicities (MUL5) are related to our most central collisions with $b=1 \mathrm{fm}$ albeit the $3 \mathrm{fm}$ collisions also populate this multiplicity region.

The intermediate mass fragment multiplicities depend strongly on the bombarding energy. The average multiplicities of intermediate mass fragments $(A>4$, without filter) is shown in Fig. 17 for the $\mathrm{Nb}+\mathrm{Nb}$ system as a function of bombarding energy, for the most central and the peripheral $(b=7 \mathrm{fm})$ collisions. Observe that for central collisions the average multiplicity $\left\langle M_{c}(A>4)\right\rangle$ is one at low energies (fusion) and has a maximum of about five to six intermediate mass fragments at intermediate energies $(E \approx 100 A \mathrm{MeV})$. This clearly demonstrates that the system completely breaks up into the lightest $(A<4)$ fragments at higher energies, as discussed in Fig. 9. This is in accordance with the quantum statistical model predictions ${ }^{51,52}$ which observe the same dependence of $M_{c}(A>4)$ on entropy and bombarding energy. This behavior should be experimentally investigated. For peripheral collisions we find at all energies a breakup into two projectile and targetlike residues. 

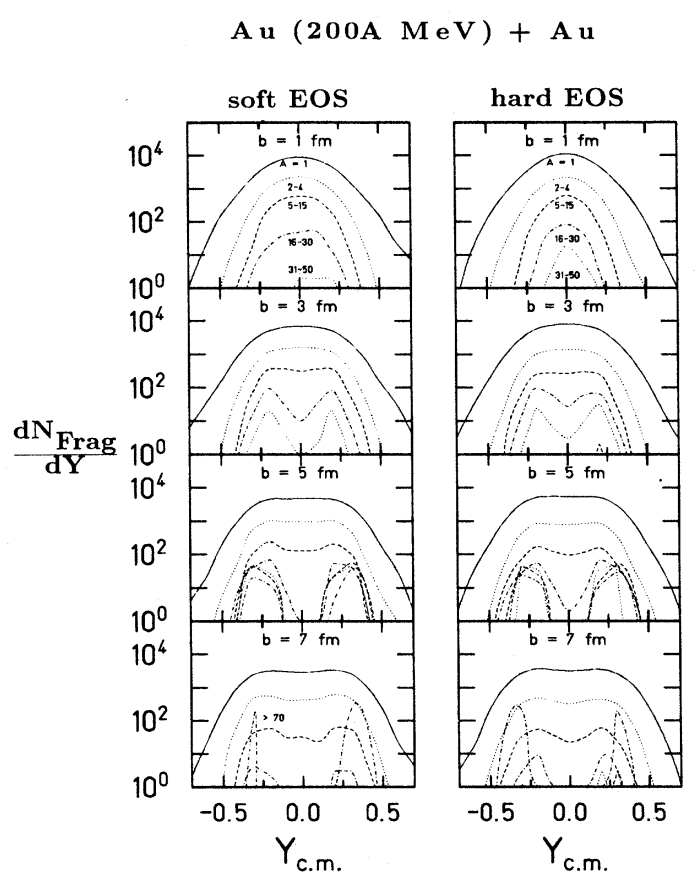

FIG. 18. Fragment rapidity distributions $d N_{\text {frag }} / d Y$ for different mass intervals from the reaction $\mathrm{Au}(200 \mathrm{~A} \mathrm{MeV})+\mathrm{Au}$ as a function of the impact parameter as indicated. The distributions obtained with the soft EOS (left column) are compared to the case of the hard EOS (right column).

\section{FRAGMENT FLOW AND THE EOS}

Let us now turn to the collective flow which can be used to study the properties of nuclear matter at high density. It has been proposed a long time ago that complex fragments should exhibit flow effects more clearly, since they are subjected to fewer $n-n$ collisions and to less random thermal motion. ${ }^{12}$ Here the dependence of collective flow effects on the fragment mass is studied for the first time in a microscopic theory.

\section{A. Rapidity distributions of the fragments - nuclear stopping as a measure of the cross section}

The longitudinal momentum distribution, to be more precise, the rapidity distribution $d N_{\text {frag }} / d Y$ of various fragment mass bins in the reaction $\mathrm{Au}(200 \mathrm{~A} \mathrm{MeV})+\mathrm{Au}$ is depicted in Fig. 18 as a function of the impact parameter. We would like to emphasize that the distinct fragment species can be related to a single source in momentum space only for the most central events $(b=1 \mathrm{fm})$. Only here all baryons have been stopped and true multifragmentation is observed (see our discussion of the liquid-vapor phase transition in the previous section). Already for $b=3 \mathrm{fm}$ a clear separation of two sources, namely the projectile and target remnants, is observed for the heavy fragments $(A>15)$. The two maxima of these curves directly reflect the finite impact parameters. Note that the $d N_{\text {frag }} / d Y$ distributions do not depend on the EOS employed.

Figure 19 depicts the bombarding energy dependence

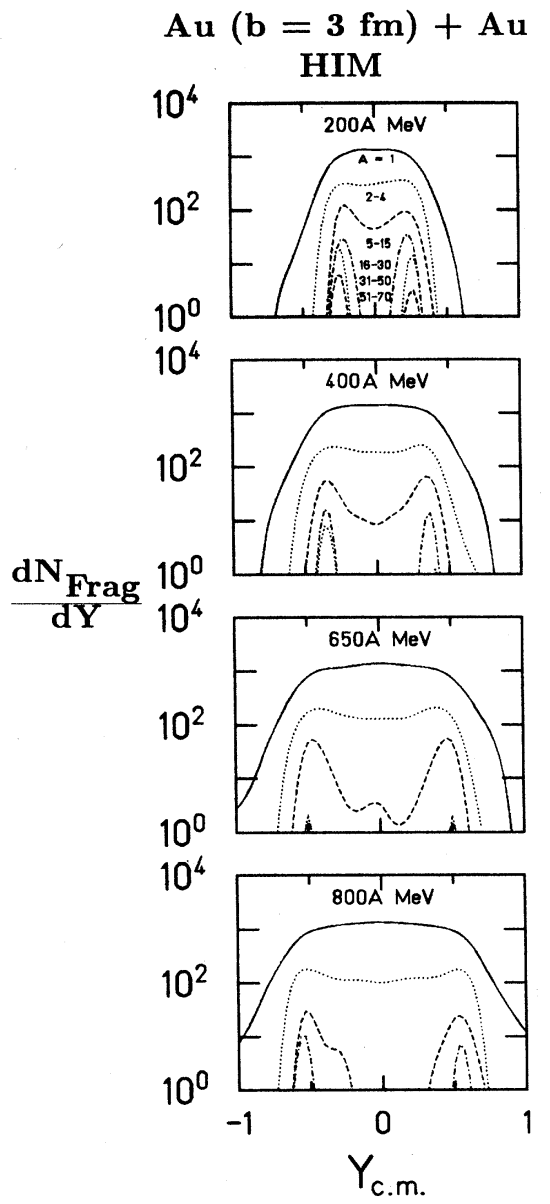

FIG. 19. Fragment rapidity distributions $d N_{\text {frag }} / d Y$ for the reaction $\mathrm{Au}(b=3 \mathrm{fm})+\mathrm{Au}$ at different bombarding energies and fragment classes as indicated. The hard local potential has been supplemented by momentum dependent potentials and effective cross sections (HIM).

of the $d N_{\text {frag }} / d Y$ distributions for the $\mathrm{Au}+\mathrm{Au}$ reaction at $3 \mathrm{fm}$ impact parameter; however, now both in medium corrected (reduced) cross sections (Refs. 36, 65, 66, 43, 24 , and 71) and the momentum dependent interactions $\mathrm{s}^{22,42}$ have been included. Note that with the $30 \%$ reduced cross sections nuclear matter is more transparent, i.e., the rapidity distributions are more forward-backward peaked. This offers the opportunity to determine the inmedium cross sections directly from the experimental $d N / d Y$ distributions. This is shown quite clearly in Fig. 20 , which compares the total rapidity distributions for the interactions S, SM, and SIM for the $200 A \mathrm{MeV}$ $\mathrm{Au}+\mathrm{Au}$ collision. One clearly observes that the local potential (equation of state) has little influence on the rapidity spectra. The distributions obtained with the interactions $H$ (HIM) are almost identical to those ones obtained with the interaction $S$ (SIM). On the other hand, the distribution obtained with and without the inmedium corrections exhibit clearly an increase of transparency when the in-medium corrections are switched on. This is most pronounced for the intermediate mass fragments and could serve as a signal to determine exper- 
total rapidity distribution

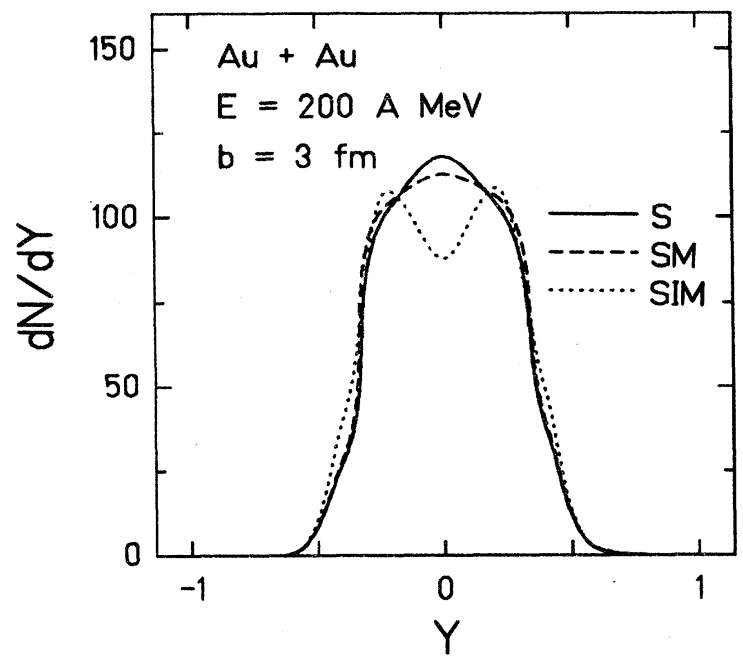

FIG. 20. Total rapidity distributions in the reaction $\mathrm{Au}$ $(200 A \mathrm{MeV}, b=3 \mathrm{fm})+\mathrm{Au}$ for the different interactions S, SM, and SIM.

imentally the effective scattering cross sections, which are related directly to the macroscopic (hydrodynamic) transport coefficients (viscosity ${ }^{73,24}$ and heat conductivity). This increased transparency is seen if one integrates over all fragments; then the total baryon rapidity distributions exhibit a single peak distribution for the interactions $S$ and SM, while the interaction SIM shows two peaks close to the rapidity of the projectile and the target. ${ }^{24,25,27}$ This indicates an incomplete stopping of the incident nuclei.

Recent $d N / d Y$ data ${ }^{71,18}$ indicate no substantial modifications of the $U-U n-n$ cross section, hence, $\sigma^{\mathrm{eff}} \approx \sigma^{\mathrm{UU}}$. This is of utmost importance, since the mag-

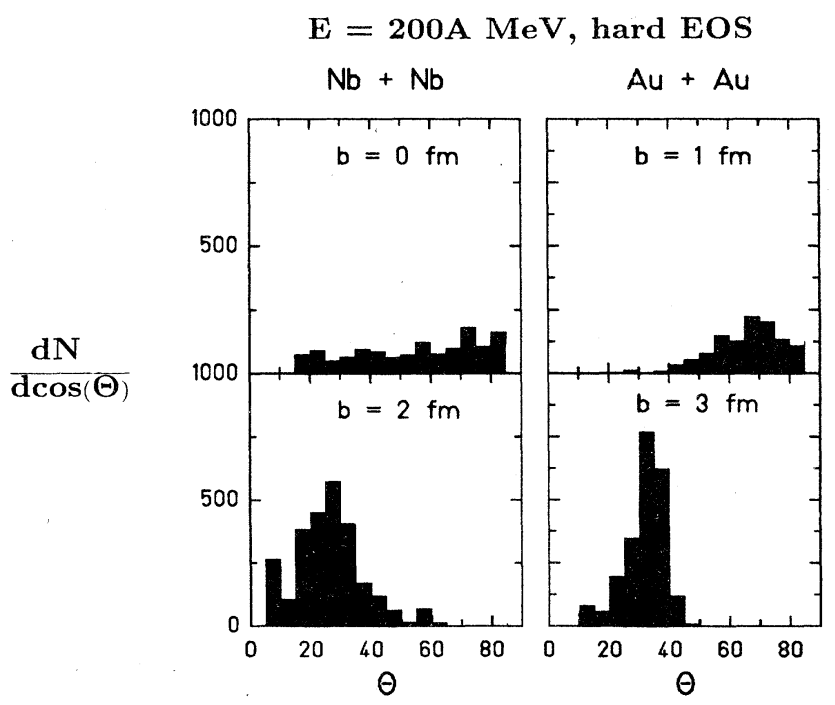

FIG. 21. Flow angle distribution $d N / d \cos \theta$ (integrated over all fragments) for the reactions $\mathrm{Nb}+\mathrm{Nb}$ (left column) and $\mathrm{Au}+\mathrm{Au}$ (right column) at $200 \mathrm{~A} \mathrm{MeV}$ bombarding energy at different impact parameters as indicated.

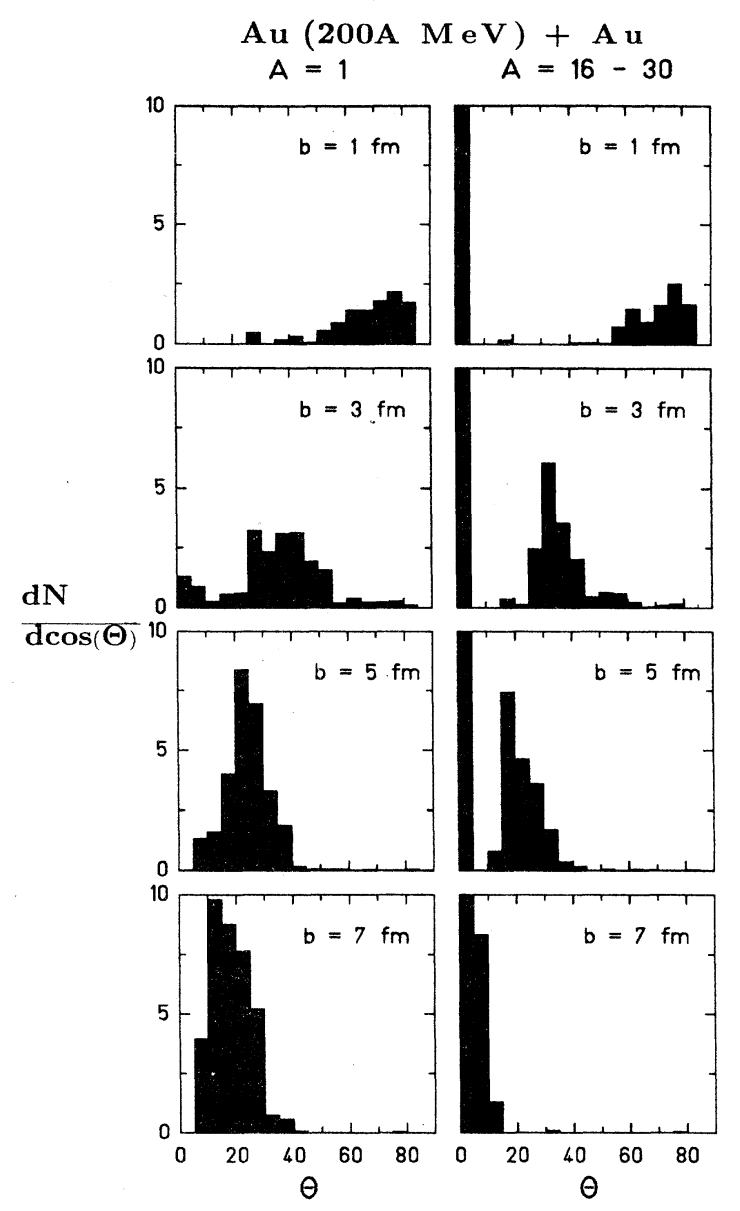

FIG. 22. Flow angle distribution $d N / d \cos \theta$ for the reaction $\mathrm{Au}(200 A \mathrm{MeV}$, hard EOS) + Au for single nucleons $(A=1$, left column) and intermediate mass fragments ( $A=16-30$, right column) as a function of the impact parameter.

nitude of the transverse flow depends sensitively on the effective scattering cross sections, as is shown below (see also Refs. 36 and 43).

\section{B. Fragment flow and transverse momentum analysis}

The dependence of the collective flow angle distribution $[d N / d \cos (\theta)]$ on the mass of projectile and target is shown in Fig. 21. The $\mathrm{Au}+\mathrm{Au}$ system exhibits larger flow angles than the $\mathrm{Nb}+\mathrm{Nb}$ system at the corresponding scaled impact parameters $\widetilde{b}=b /\left(R_{p}+R_{t}\right)$. Ideal fluid dynamics does not give such a mass dependence, ${ }^{34}$ while it is indeed found in the data., ${ }^{3,6}$ Large viscous effects must be incorporated in order to yield such a mass dependence in the nuclear fluid model. ${ }^{72}$

Now we want to study the dependence of the flow on the mass of the emitted fragments. Figure 22 shows the finite flow angles for both free nucleons and for the intermediate mass fragments in central collisions of $\mathrm{Au}+\mathrm{Au}$. The flow angles do change little-within the statistical uncertainty - when the width of the Gaussians, i.e., the effective range of the potential, is changed, or when the momentum dependent interactions are turned on. They 

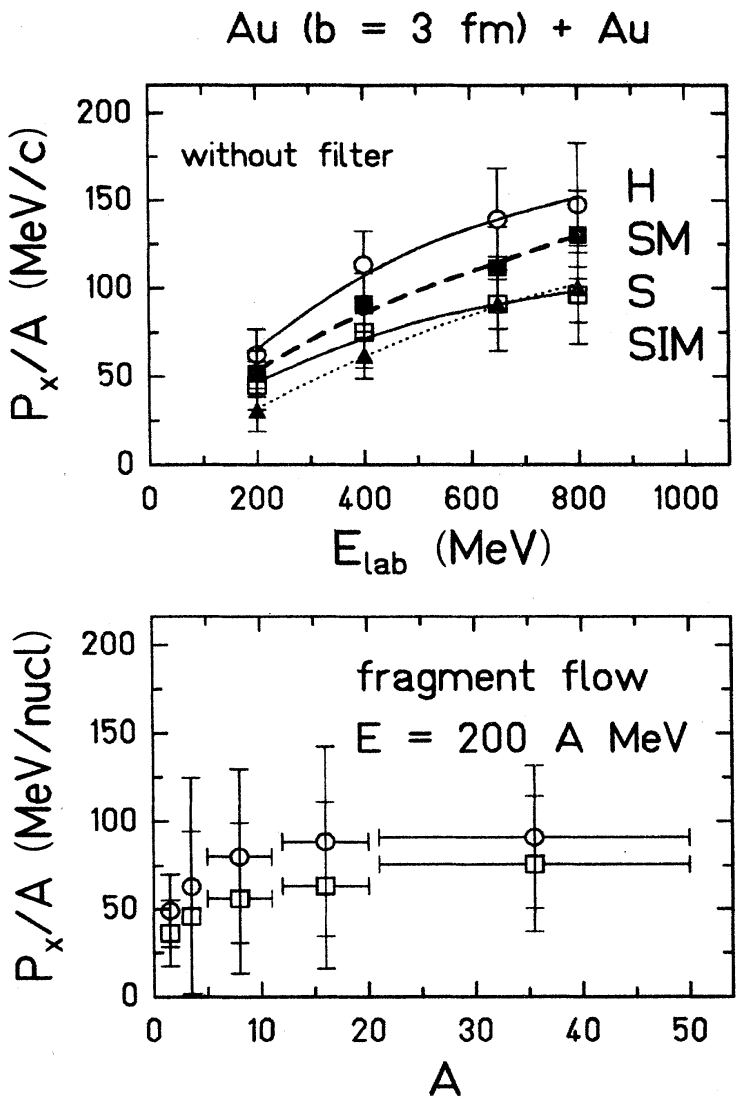

FIG. 23. Excitation function of the flow (absolute value of the $p_{x} / A-Y$ distribution at $Y=Y_{\mathrm{Pr}}$ ) for the reaction $\mathrm{Au}$ (200-800A MeV, $b=3 \mathrm{fm})+\mathrm{Au}$ obtained with the different interactions as indicated (upper part). The lower part shows the mass dependence of the fragment flow.

do drop by $30 \%$ when the in-medium corrected cross sections are employed. The flow angle $\theta$ decreases rapidly with impact parameter; for the intermediate mass fragments $(A=16-30)$ we find $\theta \approx 40^{\circ}$ at $b=3 \mathrm{fm}$. The flow vanishes if very heavy fragments $(A>30)$ are considered-these are mainly projectile and target residues.

Let us now turn to the average transverse momentum $p_{x}(y)$ (Ref. 7) in the scattering plane. It has been shown theoretically that $p_{x}(y)$ exhibits an enhanced sensitivity to the nuclear EOS as compared to other observables (Refs. 13, 14, 16, 17, 24, and 27). Here we want to concentrate on the transverse momentum carried by fragments of different mass: Figure 23 shows the excitation function of $p_{x}\left(y_{P_{r}}\right)$ for all nucleons (upper part). The dependence of $p_{x} / A$ on the mass of the fragments is shown in the lower part. Observe the increase of $p_{x} / A$ with $A_{f}$. This fragment mass dependence can also be seen in Figs. 24 and 25 which shows the rapidity dependence of $p_{x}(y)$ at $200 \mathrm{~A} \mathrm{MeV}$ for various fragment mass intervals.

We discuss these results now in detail. By its definition the transverse momentum analysis fails to detect flow effects for very central collisions; because of symmetry it
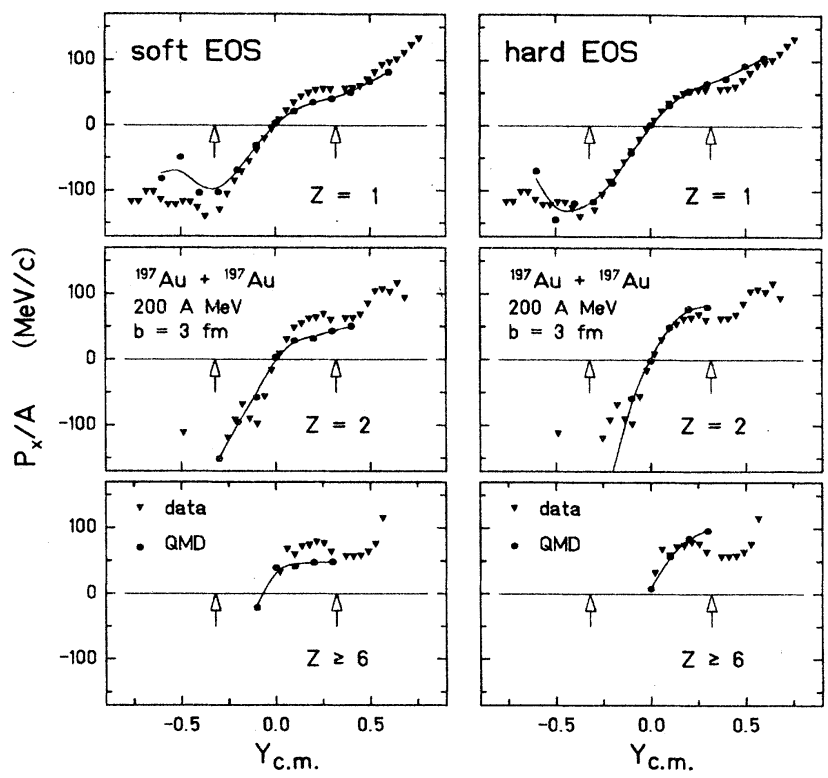

FIG. 24. The transverse momentum distributions $p_{x} / A-Y$ of light and intermediate mass fragments obtained from the QMD calculations for the reaction $\mathrm{Au}(200 A \mathrm{MeV}, b=3$ $\mathrm{fm})+\mathrm{Au}$ are compared to the data from Ref. 18. The data (triangles) have been obtained for the different fragment classes with $Z=1 \quad(A-1-2), \quad Z=2 \quad(A=3-4)$, and $6 \leq Z \leq 10$ $(12 \leq A \leq 20)$. The QMD results (circles, solid lines) have been obtained with the plastic ball filter. The left (right) column compares the results obtained from the soft (hard) EOS to the data. Both the QMD results and the data have been taken at the impact parameter (multiplicity) where the transverse momentum as a function of impact parameter (multiplicity) has a maximum (this maximum occurs at $b=3 \mathrm{fm}$ in the calculation and in the experimental MUL3 bin).

is $p_{x}(b=0 \mathrm{fm}) \equiv 0$. A clear maximum is experimentally observed in the multiplicity dependence $p_{x}\left(N_{p}\right)$ (Ref. 18) in the bins MUL3 and MUL4. The analogous maximum is observed in the calculated impact parameter dependence $p_{x}(b)$ at $b=3 \mathrm{fm} \cdot{ }^{14,24,27}$ Data and theory can thus be directly confronted by comparing the $p_{x} / A$ values at the corresponding maxima. One has to be careful about the exact definition of the collective flow: One possible definition is based on the slope of the $p_{x}-Y$ distribution at midrapidity. ${ }^{8}$ Most of the presently available data include only single nucleons and the lightest fragmentsthe intermediate mass fragments have not yet been measured with a $4 \pi$ detector except for the $200 \mathrm{~A} \mathrm{MeV}$ $\mathrm{Au}+\mathrm{Au}$ collision of Ref. 19. However, we see that the intermediate mass fragments exhibit the flow more clearly. The slope of the $p_{x} / A-Y$ distribution cannot be defined for such fragments, because their yields are strongly peaked at the projectile and target rapidity (see Fig. 18). Only a few fragments are found at midrapidity, except for the most central collisions $(b \leq 1)$, where the $p_{x} / A$ values are small. There is only one way out of this situation, namely to study the $p_{x} / A$ values at the maximum yield, i.e., close to the projectile rapidity $Y_{P r} \cdot{ }^{17,12}$

Figure 23 shows the excitation function of the flow of 

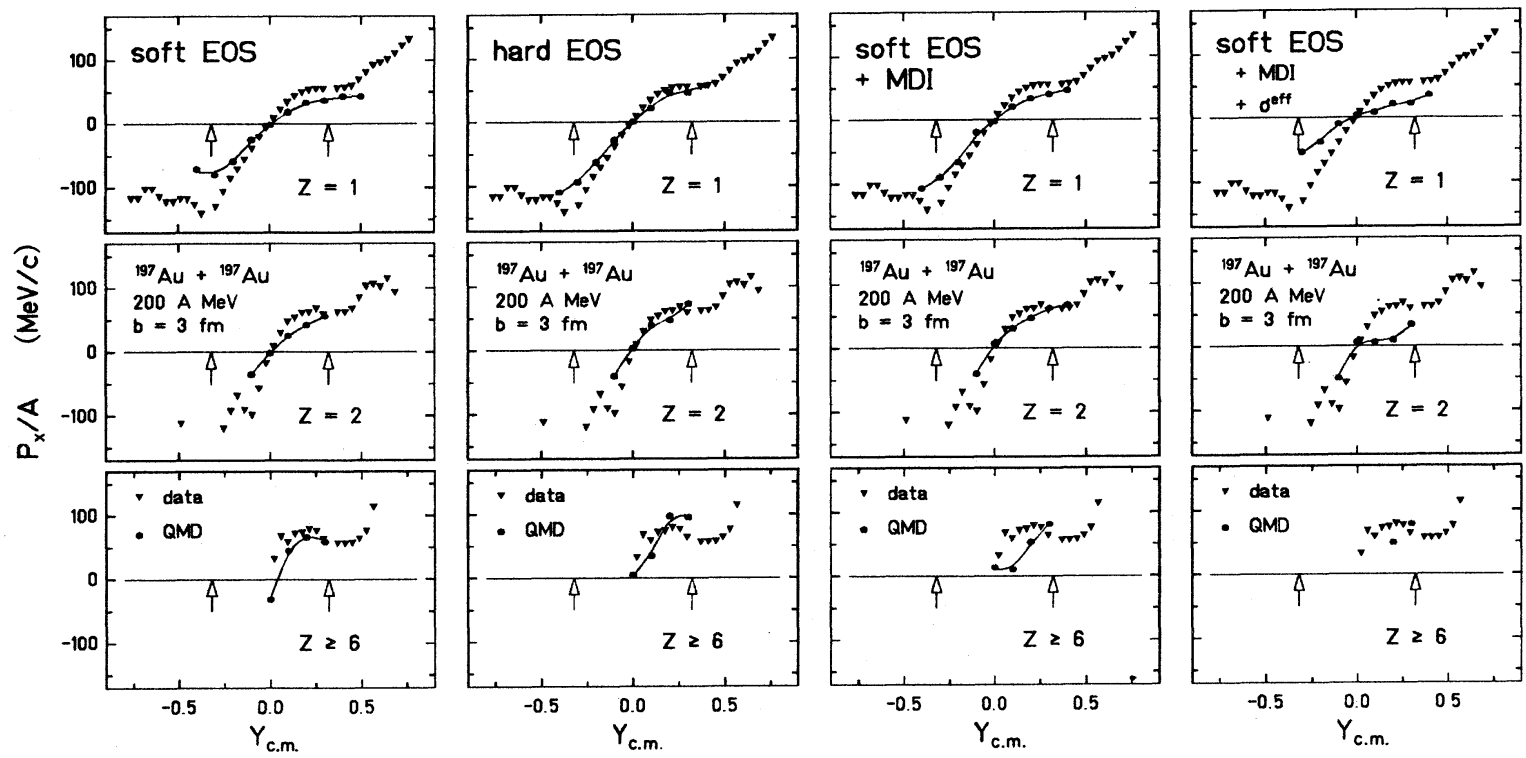

FIG. 25. Same as Fig. 24, but the width of the Gaussians has been switched from $L=2.1 \mathrm{fm}$ to $L=1.5 \mathrm{fm}$. The left two columns can be directly compared to Fig. 24 to see the moderate influence of $L$.

all nucleons (upper part) for the reaction $\mathrm{Au}$ $(E=200-800 A \mathrm{MeV}, b=3 \mathrm{fm})+\mathrm{Au}$ for the hard $(H)$, the soft $(S)$, the soft EOS plus the momentum dependent interactions (SM), and for the momentum dependent interactions with the reduced cross sections (SIM). One observes at all energies a clear difference between the hard and the soft EOS. The transverse momentum of the free nucleons obtained with the hard EOS exceeds those obtained with the soft EOS by approximately $40 \%$.

This difference between the hard and the soft local potential is not changed, no matter whether the purely local interactions $H$ and $S$, or the in medium corrected interactions HIM and SIM are used. ${ }^{25}$ The absolute values of the flow drops by a factor of about 2 when the reduced $^{65,66}$ cross sections are employed. The third curve in the upper part of Fig. 23 shows the excitation function of the flow obtained with the soft EOS plus the additional momentum dependent interactions (SM). In the lowenergy regime $(E=200 \mathrm{~A} \mathrm{MeV})$ one observes little difference between the cases $S$ and SM, while for the high-energy regime $(E>200 A \mathrm{MeV})$ the additional repulsion of the MDI increase the transverse flow. There is still a clear difference of about one standard deviation between the soft EOS with MDI (SM) and the hard EOS without MDI $(H)$.

The lower part of Fig. 23 shows the mass dependence of the fragment flow at beam rapidity. Note that the flow of the intermediate mass fragments is more pronounced than that of the free nucleons.

We now proceed to compare directly the data for the $\mathrm{Au}+\mathrm{Au}$ reaction at $200 A \mathrm{MeV}$ bombarding energy ${ }^{18,19}$ to the theoretical results obtained with the soft $(S)$ and the hard $(H)$ EOS (without medium corrections), respectively. Figure 24 shows the calculated (solid circles) and the measured (solid triangles) in-plane transverse momenta per nucleon $p_{x} / A$ versus rapidity for the reaction $\mathrm{Au}$
(200A MeV, $b=3 \mathrm{fm})+\mathrm{Au}$, evaluated with the plastic ball efficiency cuts for different fragment masses and for the soft and the hard EOS, respectively. The corresponding calculated $d N_{\text {frag }} / d Y$ distributions have been shown in Fig. 18. For $b=3 \mathrm{fm}$, where the maximal $p_{x} / A$ values occur, the heavier fragment yields $(A>10)$ are peaked at projectile and target rapidity $(Y \approx \pm 0.3)$. The light fragment distributions ( $A \leq 4)$ exhibit a broad maximum at $Y_{\text {c.m. }}=0$.

Note the increase of the $p_{x} / A$ values with the mass of the fragments both in theory and experiment and the dependence of the $p_{x} / A$ values on the equation of state. This difference is most significant for the intermediate mass fragments $(A \geq 6)$. These fragments are formed at early times in the reaction as a result of the shock wave traveling through the interpenetrating projectile. The heaviest fragments $(A>30)$, on the other hand, are formed in a decay chain from the excited projectile and target residue at late times. Hence, they do not carry the strong signatures of the compression stage.

The measured transverse momenta $p_{x} / A$ were obtained for the MUL3 bin, ${ }^{18,19}$ where again $p_{x}\left(N_{p}\right)$ is maximal, for fragments with $Z=1, Z=2$, and $Z=6-10$.

In order to draw conclusions from the comparison of the present calculations with the data the same filter is applied, which has been used above to evaluate the multiplicity distributions. The left (right) column shows the $p_{x} / A$ distributions for fragments with $Z=1,(A=1,2)$, $Z=2(A=3,4)$, and $Z \geq 6(A=12-20)$ obtained with the soft (hard) EOS (solid circles) as compared to the data (triangles). We would like to point out that the soft EOS underpredicts the data. The right column shows that the agreement with the data is improved for all fragment masses if the hard EOS is employed in the QMD calculation.

We would like to stress again that the QMD calcula- 
tions are obtained for the impact parameter $b=3 \mathrm{fm}$ for which the maximum of the $p_{x}(b) / A$ distribution is found. The data are taken from the multiplicity bin for which the maximum transverse flow is observed (MUL3). If we make use of this implicit relation between impact parameter and multiplicity, we conclude that the EOS is quite hard at $\rho / \rho_{0} \approx 2$, which is the maximum density found at these rather moderate bombarding energies.

All the results of Fig. 24 were obtained with $L=2.1$ fm. In order to study the influence of the effective range of our interaction on the $p_{x}$ distribution we present in Fig. 25 the $p_{x}-Y$ distributions for the hard and soft local potential and also for the soft local potential plus the different in-medium corrections obtained with $L=1.5$ fm.

Note that the inclusion of the momentum dependent interaction leads to an increase of $p_{x} / \boldsymbol{A}$. Reduced cross sections, however, lead to a strong reduction of the flow. We would like to emphasize again that the effective cross sections should be determined consistently in experiments which simultaneously measure the collective flow and baryon rapidity distribution $d N / d Y$, i.e., different projections of the triple differential cross section. ${ }^{12}$

Reduced in-medium cross sections would only be balanced by an even harder EOS. Large $p_{x}$ values could also be created, if the effective cross sections were increased as compared to the UU cross section, which would be opposite to current theoretical understanding. ${ }^{65,66}$ Increased in-medium cross sections, e.g., due to precritical scattering in the vicinity of a phase transition, would be a most fascinating resolution of the puzzle about the stiffness of the EOS from supernova explosions and high-energy heavy ion collisions. However, at moderate energies, $E_{\text {lab }} \sim 100 \mathrm{MeV} /$ nucleon, the increase of the $p n$ cross section (as compared to the $p p$ cross section used in the Cugnon parametrization employed here and in all other VUU calculations ${ }^{39-43}$ ) also causes an increase of the isospin averaged cross section and therefore an increase of the flow $\left(p_{x}\right)$ values.

Hence, we conclude that a systematic measurement of the flow and the $d N_{\text {frag }} / d Y$ distribution of complex fragments is urgently needed to open up the possibility to pin down the nuclear matter properties at the higher bombarding energies.

\section{SUMMARY}

In the present paper we have studied aspects of multifragmentation in high-energy heavy ion collisions and the dependence of the collective flow of nucleons and intermediate mass fragments on momentum dependent interactions, in-medium corrections of the scattering cross sections and on the nuclear EOS.

The quantum molecular dynamic (QMD) approach has been employed to simulate heavy ion collisions on a microscopic level. This model can describe multifragmentation because of the explicit treatment of many-body correlations. The fragment multiplicities depend only negligibly on the stiffness of the EOS, but they are sensitive to the effective scattering cross sections.
We have shown that the observed power law dependence of the measured inclusive fragment yields is of geometrical origins and cannot be interpreted directly as a signal for a liquid-vapor phase transition. Impact parameter selected distributions need to be measured before a possible liquid-vapor phase transition can be studied.

The relative yields of the light fragments $(A \leq 4)$ show a strong impact parameter and bombarding energy dependence. The $d_{\text {like }} / p_{\text {like }}$ values increase with decreasing impact parameter and decreasing bombarding energy, in agreement with recent data, ${ }^{70}$ also the predictions of complex fragment multiplicities $(A>4)$ are supported by the experiment. ${ }^{18,19}$

We have then studied the influence of the momentum dependent interactions and the reduced effective nucleon-nucleon scattering cross sections on the flow in heavy ion collisions. It has been shown that the transverse momentum transfer depends on the momentum dependent potential, which tend to increase $p_{x} / A$, as well as on the reduction of the scattering cross sections which decrease $p_{x} / A$ at all energies.

However, it is shown that the longitudinal momentum distributions, i.e., the rapidity distributions $d N / d Y$ of all baryons and of the fragments are insensitive to the EOS and to the MDI, while $d N / d Y$ depends strongly on the effective cross sections. They can therefore be used to determine this important quantity, i.e., $\sigma_{\text {in-medium. }}^{\text {eff }}$ Let us note again that $\sigma$ is inversely related to the nuclear viscosity $\eta$.

Recent experiments have shown that the UehlingUhlenbeck cross sections reproduce the $d N / d Y$ data very well. Reduced cross sections lead to too much transparency, as compared to the experimental results.

The transverse momentum transfer depends at all energies on the EOS. The intermediate mass fragments are particularly sensitive on the EOS.

The fragment flow is shown to increase with fragment mass. This increase is also found experimentally.

The measured $p_{x} / A$ values for $\mathrm{Au}(200 A \mathrm{MeV})+\mathrm{Au}$ reaction exceed the $p_{x} / A$ values calculated in the present model even if a momentum dependent interaction and/or in-medium corrected cross sections are implemented, as long as the soft EOS is employed. The fragment flow data can be reproduced with the QMD model if the Uehling-Uhlenbeck cross section and the hard EOS are employed.

Increased in-medium cross sections, e.g., due to precritical scattering in the vicinity of a phase transition, would be a most fascinating resolution of the puzzle about the stiffness of the EOS from supernova explosions and high-energy heavy ion collisions. A systematic measurement of the flow and the $d N_{\text {frag }} / d Y$ distribution of complex fragments is urgently needed to open up the possibility to pin down the nuclear matter properties at the higher bombarding energies.

This work has been funded in part by the German Federal Minister for Research and Technology (BMFT) under the Contact Nos. 06 OF 772, 06 HD 776, and by the Gesellschaft für Schwerionenforschung (GSI). 
${ }^{1}$ E. Baron, J. Cooperstein, and S. Kahana, Phys. Rev. Lett. 55, 126 (1985).

${ }^{2}$ E. Baron, H. A. Bethe, G. E. Brown, J. Cooperstein, and S. Kahana, Phys. Rev. Lett. 59, 736 (1987).

${ }^{3}$ H. A. Gustafsson, H. H. Gutbrod, B. Kolb, H. Löhner, B. Ludewigt, A. M. Poskanzer, T. Renner, H. Riedesel, H. G. Ritter, A. Warwich, F. Weik, and H. Wieman, Phys. Rev. Lett. 52, 1590 (1984).

${ }^{4}$ R. E. Renfordt, D. Schall, R. Bock, R. Brockmann, J. W. Harris, A. Sandowal, R. Stock, H. Ströbele, D. Bangert, W. Rauch, G. Odyniec, H. G. Pugh, and L. S. Schröder, Phys. Rev. Lett. 53, 763 (1984).

${ }^{5}$ D. Beauvis, S. Y. Chu, S. Y. Fung, W. Gorn, A. Huie, D. Keane, J. J. Liu, R. T. Poe, B. C. Shen, and G. VanDalen, Phys. Rev. C 27, 2443 (1983); D. Beavis et al., Phys. Rev. Lett. 54, 1652 (1985); D. Beavis et al., Phys. Rev. C 33, 113 (1986).

${ }^{6}$ H. G. Ritter, K. G. R. Doss, H. A. Gustafsson, H. H. Gutbrod, K. H. Kampert, B. Kolb, H. Löhner, B. Ludewigt, A. M. Poskanzer, A. Warwick, and H. Wieman, Nucl. Phys. A447, 3c (1985).

${ }^{7}$ P. Danielewicz and G. Odyniec, Phys. Lett. 157B, 146 (1985).

${ }^{8}$ K. G. R. Doss, H. A. Gustafsson, H. H. Gutbrod, K. H. Kampert, B. Kolb, H. Löhner, B. Ludewigt, A. M. Poskanzer, H. G. Ritter, H. R. Schmidt, and H. Wieman, Phys. Rev. Lett. 57, 302 (1986).

${ }^{9}$ P. Danielewicz, H. Ströbele, G. Odyniec, D. Bangert, R. Bock, R. Brockmann, J. W. Harris, H. G. Pugh, W. Rauch, R. E. Renfordt, A. Sandowal, D. Schall, L. S. Schroeder, and R. Stock, GSI Report 87-74 (1987).

${ }^{10}$ W. Scheid, H. Müller, and W. Greiner, Phys. Rev. Lett. 32, 741 (1974).

${ }^{11}$ H. G. Baumgardt, J. U. Schott, Y. Sakamoto, E. Schopper, H. Stöcker, J. Hofmann, W. Scheid, and W. Greiner, Z. Phys. A273, 359 (1975).

${ }^{12}$ H. Stöcker, J. A. Maruhn, and W. Greiner, Phys. Rev. Lett. 44, 725 (1980); H. Stöcker et al., ibid. 47, 1807 (1981); H. Stöcker et al., Phys. Rev. C 25, 1873 (1982); G. Buchwald et al., Phys. Rev. Lett. 52, 1594 (1984).

${ }^{13}$ H. Stöcker and W. Greiner, Phys. Rep. 137, 277 (1986).

${ }^{14}$ J. J. Molitoris, J. B. Hoffer, H. Kruse, and H. Stöcker, Phys. Rev. Lett. 53, 899 (1984).

${ }^{15}$ H. Kruse, B. V. Jacak, and H. Stöcker, Phys. Rev. Lett. 54, 289 (1985).

16J. J. Molitoris and H. Stöcker, Phys. Rev. C 32, 346 (1985); Phys. Lett. 162B, 47 (1985).

${ }^{17}$ J. J. Molitoris, H. Stöcker, and B. L. Winer, Phys. Rev. C 36, 220 (1987).

${ }^{18}$ K. H. Kampert, Ph.D. thesis, University of Münster, 1986.

${ }^{19}$ K. G. R. Doss, H.-A. Gustafsson, H. Gutbrod, J. W. Harris, B. W. Jacak, K.-H. Kampert, B. Kolb, A. M. Poskanzer, H.G. Ritter, H. R. Schmidt, L. Teitelbaum, M. Tincknell, S. Weiss, and H. Wiemann, Phys. Rev. Lett. 59, 2720 (1987).

20J. Aichelin and H. Stöcker, Phys. Lett. B176, 14 (1986).

${ }^{21}$ A. Rosenhauer, J. Aichelin, H. Stöcker, and W. Greiner, J. Phys. (Paris) C4, 395 (1986).

22J. Aichelin, A. Rosenhauer, G. Peilert, H. Stöcker, and W. Greiner, Phys. Rev. Lett. 58, 1926 (1987).

${ }^{23}$ G. Peilert, A. Rosenhauer, J. Aichelin, H. Stöcker, and W. Greiner, Mod. Phys. Lett. A 3, 459 (1988).

${ }^{24}$ A. Rosenhauer et al., Phys. Rev. C (to be published).

${ }^{25}$ G. Peilert, Ph.D. thesis, University Frankfurt, 1988.

${ }^{26}$ J. Aichelin, G. Peilert, A. Bohnet, A. Rosenhauer, H. Stöcker, and W. Greiner, Phys. Rev. C 37, 2451 (1988).
${ }^{27}$ A. Rosenhauer, Ph.D. thesis, University Frankfurt, 1988.

${ }^{28} \mathrm{~K}$. Nomoto and M. Hashimoto (unpublished).

${ }^{29}$ S. E. Woosley and T. A. Weaver, Bull. Am. Astron. Soc. 16, 971 (1984).

${ }^{30}$ W. Hillebrandt, Astron. Astrophys. 110, L3-L6 (1982).

${ }^{31}$ J. R. Wilson, in Numerical Astrophysics, edited by J. Centrella, J. LeBlanc, and R. Bowers (Jones \& Bartlett, Boston, 1985); H. A. Bethe and J. R. Wilson, Astrophys. J. 295, 14 (1985).

${ }^{32}$ N. K. Glendenning, Z. Phys. A327, 295 (1987).

${ }^{33}$ A. R. Bodmer and C. N. Panos, Phys. Rev. C 15, 1342 (1977); 22, 1023 (1980).

${ }^{34}$ B. Schürmann and $W$. Zwermann, Phys. Lett. 158B, 366 (1985); Phys. Rep. 147, 1 (1987).

${ }^{35}$ T. L. Ainsworth, E. Baron, G. E. Brown, J. Cooperstein, and M. Prakash, Nucl. Phys. A464, 740 (1987)

${ }^{36}$ M. Gyulassy, K. A. Frankel, and H. Stöcker, Phys. Lett. 110B, 185 (1982). This paper, as well as the present work, employs the energy and angular-dependent $p$ - $p$ cross sections as parametrized by Cugnon et al., Phys. Rev. C 22, 1885 (1980).

${ }^{37}$ M. Gyulassy and W. Greiner, Ann. Phys. 109, 485 (1977).

${ }^{38}$ H. Kruse, B. V. Jacak, J. J. Molitoris, G. D. Westfall, and H. Stöcker, Phys. Rev. C 31, 1770 (1985).

${ }^{39}$ G. F. Bertsch, H. Kruse, and S. Das Gupta, Phys. Rev. C 29, 673 (1984).

${ }^{40} \mathrm{~J}$. Aichelin and H. Stöcker, Phys. Lett. 163, 59 (1985).

${ }^{41}$ J. Aichelin and G. F. Bertsch, Phys. Rev. C 31, 1730 (1985).

${ }^{42}$ C. Gale, G. F. Bertsch, and S. Das Gupta, Phys. Rev. C 35, 1666 (1987).

${ }^{43}$ G. F. Bertsch, W. G. Lynch, and M. B. Tsang, Phys. Lett. B189, 384 (1987).

${ }^{44}$ C. Gregoire, B. Remaud, F. Sebille, and L. Vinet, Nucl. Phys. A465, 317 (1987).

${ }^{45}$ R. W. Minich, S. Agarwal, A. Bujak, J. Chuang, J. E. Finn, L. J. Gutay, A. S. Hirsch, N. T. Porile, R. P. Scharenberg, B. C. Stringfellow, and F. Turkot, Phys. Lett. 118B, 458 (1982).

${ }^{46}$ J. E. Finn, S. Agarwal, A. Bujak, J. Chuang, L. J. Gutay, A. S. Hirsch, R. W. Minich, N. T. Porile, R. P. Scharenberg, B. C. Stringfellow, and F. Turkot, Phys. Rev. Lett. 49, 1321 (1982).

${ }^{47}$ A. S. Hirsch, A. Bujak, J. E. Finn, L. J. Gutay, R. W. Minich, N. T. Porile, R. P. Scharenberg, B. C. Stringfellow, and F. Turkot, Phys. Rev. C 29, 508 (1984).

${ }^{48}$ A. D. Panagiotou, M. W. Curtin, H. Toki, D. K. Scott, and P. J. Siemens, Phys. Rev. Lett. 52, 496 (1984).

${ }^{49}$ L. Csernai and J. Kapusta, Phys. Rep. 131, 223 (1986).

${ }^{50}$ B. Jakobsson, G. Jönsson, B. Lindkvist, and A. Oskarsson, Z. Phys. A307, 293 (1982).

${ }^{51}$ H. Stöcker, G. Buchwald, G. Graebner, P. Subramanian, J. A. Maruhn, W. Greiner, B. V. Jacak, and G. D. Westfall, Nucl. Phys. A400, 63 (1983).

${ }^{52}$ D. Hahn and H. Stöcker, Nucl. Phys. A476, 718 (1988).

${ }^{53}$ A. I. Warwick, H. Wieman, H. H. Gutbrod, M. R. Maier, J. Peter, H. G. Ritter, H. Stelzer, F. Weik, M. Freedman, D. J. Henderson, S. B. Kaufman, E. P. Steinberg, and B. D. Wilkins, Phys. Rev. C 27, 1083 (1983).

${ }^{54}$ G. Fai and J. Randrup, Nucl. Phys. A404, 551 (1983).

${ }^{55} \mathrm{X}$. Campi, J. Desbois, and E. Lipparini, Phys. Lett. 142B, 8 (1984).

56J. P. Bondorf, R. Donangelo, I. N. Mishustin, C. J. Petrick, H. Schulz, and K. Sneppen, Nucl. Phys. A443, 347 (1985); A444, 460 (1985).

${ }^{57}$ L. Wilets, Y. Yariv, and R. Chestnut, Nucl. Phys. A282, 359 (1977); C. Dorso, S. Duarte, and J. Randrup, Phys. Lett. B 188, 287 (1987). 
${ }^{58}$ S. M. Kiselev and Y. E. Prokovskil, Yad. Fiz. 38, 82 (1983) [Sov. J. Nucl. Phys. 38, 46 (1983)].

${ }^{59}$ T. J. Schlagel and V. R. Pandharipande, Phys. Rev. C 36, 192 (1987).

${ }^{60}$ G. E. Beauvais, D. H. Boal, and J. C. K. Wong, Phys. Rev. C 35, 545 (1987).

${ }^{61}$ S. M. Kiselev, Yad. Fiz. 41, 94 (1985) [Sov. J. Nucl. Phys. 41, $58(1985)]$.

62 J. Knoll and B. Strack, Phys. Lett. 149B, 45 (1984).

${ }^{63}$ W. Bauer, G. F. Bertsch, and S. Das Gupta, Phys. Rev. Lett. 58, 863 (1987).

${ }^{64}$ S. Das Gupta, C. Gale, J. Gallego, H. H. Gan, and R. D. Ratna Raju, Phys. Rev. C 35, 556 (1987).

${ }^{65}$ B. ter Haar, R. Malfliet, and W. Botermans, Phys. Rep. 149, 207 (1987); Phys. Lett. 172B, 10 (1986).

${ }^{66} \mathrm{~J}$. Cugnon, A. Lejeunne, and P. Grange, Phys. Rev. C 35, 861 (1987).

${ }^{67}$ D. R. Bowman, R. J. Charity, R. J. McDonald, M. A. McMahan, G. J. Wozniak, L. G. Moretto, W. L. Kehoe, S.
Bradley, A. C. Mignerey, A. Moroni, A. Bracco, I. Iori, and M. N. Namboodiri, Phys. Lett. B189, 282 (1987).

${ }^{68}$ P. J. Siemens and L. J. Kapusta, Phys. Rev. Lett. 43, 1486 (1979).

${ }^{69}$ G. Bertsch and J. Cugnon, Phys. Rev. C 24, 2514 (1981).

${ }^{70}$ K. G. R. Doss et al., Phys. Rev. C 32, 116 (1985); 37, 163 (1988).

${ }^{71}$ D. Keane, S. Y. Chu, S. Y. Fung, Y. M. Liu, L. J. Qiao, G. VanDalen, M. Vient, S. Wang, J. J. Molitoris, and H. Stöcker, Phys. Rev. C (to be published); Proceedings of the 8th High Energy Heavy Ion Study, LBL Berkeley, 1987 (unpublished).

${ }^{72}$ T. Rentzsch, Ph.D. thesis, University Frankfurt, 1988; T. Rentzsch, J. A. Maruhn, H. Stöcker, and W. Greiner, Proceedings of the XVI International Conference on Gross Properties of Nuclei and Nuclear Excitations, Hirschegg, Austria, 1988 (unpublished).

${ }^{73}$ B. Schürmann and W. Zwermann, Phys. Rev. Lett. 59, 2848 (1987). 